\title{
Deep-inelastic final states in a space-time description of shower development and hadronization
}

\author{
John Ellis* \\ Theoretical Physics Division European Organization for Nuclear Research (CERN), CH-1211 Geneva 23, Switzerland \\ Klaus Geiger ${ }^{\dagger}$ \\ Physics Department, Brookhaven National Laboratory, Upton, New York 11973 \\ H. Kowalski ${ }^{\ddagger}$ \\ Deutsches Elekrunen-Synchrotron DESY, Notkestrasse 85, 22607 Hamburg, Germany
}

(Received 29 May 1996)

\begin{abstract}
We extend a quantum kinetic approach to the description of hadronic showers in space, time, and momentum space to deep-inelastic ep collisions, with particular reference to experiments at DESY HERA. We follow the history of hard scattering events back to the initial hadronic state and forward to the formation of colorsinglet prehadronic clusters and their decays into hadrons. The time evolution of the spacelike initial-state shower and the timelike secondary partons are treated similarly, and cluster formation is treated using a spatial criterion motivated by confinement and a nonperturbative model for hadronization. We calculate the time evolution of particle distributions in rapidity, transverse, and longitudinal space. We also compare the transverse hadronic energy flow and the distribution of observed hadronic masses with experimental data from HERA, finding encouraging results, and discuss the background to large-rapidity-gap events. The techniques developed in this paper may be applied in the future to more complicated processes such as $e A, p p, p A$, and $A A$ collisions. [S0556-2821(96)00721-7]
\end{abstract}

PACS number(s): 12.38.Aw, 12.38.Qk, 13.60.-r, 13.87.-a

\section{INTRODUCTION}

The short-distance physics of isolated hard partonic processes in high-energy particle collisions is nowadays generally well understood within perturbative $\mathrm{QCD}$, either by calculating matrix elements with parton final states, or by parton shower evolution based on the QCD renormalization group equation. On the other hand, the long-distance dynamics of nonperturbative soft processes and of the confinement mechanism in the process of final-state parton-hadron conversion is presently not calculable from first principles, and, therefore, requires phenomenological model building. Nevertheless, over the past two decades, the combination of perturbative QCD calculus and realistic modeling of the nonperturbative physics has been developed sufficiently to provide an impressively accurate and predictive description of a large class of experimental observables connected with largemomentum jets [1-3].

However, with the advent of the DESY $e p$ collider HERA ( $e p$, possibly $e A$ ) and the Fermilab Tevatron $(p \bar{p})$, a new regime of QCD at high parton density is opening up, with which one is just beginning to come to grips. This regime will be further explorable with the future accelerators, the BNL Relativistic Heavy Ion Collider (RHIC) $(A A)$ and CERN Large Hadron Collider (LHC) ( $p p, p A, A A)$. The common novel feature of these machines is the opportunity to study the production and evolution of a system of a large

\footnotetext{
*Electronic address: John.Ellis@cern.ch

${ }^{\dagger}$ Electronic address: klaus@bnl.gov

‡Electronic address: kowalski@desy.de
}

number of partons per unit phase space $\Delta \Omega \equiv \Delta r \Delta k$, which provides a possible source for new phenomena such as nontrivial statistical particle correlations, coherence and interference effects, dissipation, and collective excitations. Examples of the experimental manifestation of such phenomena are: in $e p(e A)$ collisions, an enhanced growth of the parton distributions at small Bjorken $x$ [4], as well as the observation of diffractive events with large rapidity gaps between target and current fragmentation regions [5,6]; in $e A, p A$, $A A$ collisions, events with multiple parton scattering $[7,8]$, the QCD Landau-Pomeranchuk-Migdal effect [9], and jet quenching [10]; in $A A$ collisions, the possible formation of a high-temperature, deconfined parton plasma [11].

To quantify what we mean by high parton density, consider the hard interaction of a probing particle with a hadron or nucleus via a momentum transfer $Q \gg \Lambda_{\mathrm{QCD}}$. The probe can be, e.g., a photon (in deep-inelastic scattering) or a parton (in hadronic or nuclear collisions). The hard interaction probes space-time distances, $r \sim 1 / Q$, thereby resolving a density of partons in the probed hadron (nucleon) or nucleus which may be characterized by the number of quark and gluon quanta with a definite value of rapidity, $y \simeq \ln (1 / x)$, in the transverse plane, $\rho_{q g} \propto R_{N}^{-2} d N_{q g} / d y \simeq$ $R_{N}^{-2}\left[A x f_{N}\left(x, Q^{2}\right)\right]$, where $f_{N}\left(x, Q^{2}\right)$ denotes the sum of quark and gluon parton distributions in a nucleon, $R_{N}$ the nucleon radius, and $A$ the number of nucleons. One can distinguish three regions [12]: (i) $r \ll 1 \mathrm{fm}, \rho_{q g} \ll R_{N}^{-2}$, the short-distance, low-density regime of perturbative QCD, (ii) $r \approx 1 \mathrm{fm}$, the nonperturbative QCD domain of the complex mechanism of confinement; and (iii) $r \ll 1 \mathrm{fm}, \rho_{q g} \gtrsim R_{N}^{-2}$, the high-density regime where a dense parton system is probed 
at short distances, so that perturbative methods may be applied, within a statistical approach.

There are two extreme ways to penetrate a system of partons with large density at short space-time distances: one way is deep-inelastic ep scattering $(A=1)$ at high energy in the region of very small Bjorken $x \ll 1$. For instance, at HERA, the extrapolation of experimental data implies 30-40 gluons in a proton at $x \simeq 10^{-4}$ [13]. The other way is through collisions of heavy nuclei, in which one can reach high parton densities at not so very high energies or small $x$, due to the large number of overlapping nucleons $(A \gg 1)$. This presumably can be achieved at RHIC $\left(x \approx 10^{-1}-10^{-2}\right)$, and certainly at the LHC $\left(x \approx 10^{-3}-10^{-4}\right)$. In particular, at the LHC both the conditions of small $x$ and large $A$ may be combined. It is clear that the theoretical study of highdensity QCD requires the development of new methods by recruiting techniques from relativistic many-body physics, the kinetic theory of transport phenomena, renormalization group at finite density (and finite temperature), etc.

The purpose of the present paper is to start looking at this physics from a space-time point of view, and to study the dynamics of high parton densities in deep-inelastic ep scattering (DIS) in the kinematical region covered by the HERA experiments ZEUS and H1. In the light of the detailed hadronic measurements at HERA, which provide information about the underlying parton and hadron dynamics, our emphasis is the study of the interplay between perturbative partonic processes and nonperturbative hadron formation. We employ a kinetic space-time approach to parton-shower evolution combined with a statistical model of parton-hadron conversion [14] that allows us to follow the time development of the particle system in both momentum space and position space, i.e., in seven-dimensional phase space $d^{3} r d^{4} k$.

The space-time structure of the production and evolution of partonic color charges and their conversion into "white" hadrons is the key problem in the dynamics of complex multi-parton systems. In the context of high-density QCD, insight into this problem is especially important, because the presence of many partons close by in phase space, generated by the particle dynamics itself, necessarily causes the propagation and interaction of quanta to become nonlocal and to be correlated statistically in position space and color space. As the system evolves, these conditions will change with time and will, in general, depend on the local density of particles. This is to be contrasted with the familiar translation-invariant evolution of well-separated parton jets in empty space, in which case space-time correlations are absent or irrelevant, because the jets evolve undisturbed by one another. An interesting example of a deviation from unscathed jet evolution has been conjectured to occur in $e^{+} e^{-}$annihilation into hadrons via $W^{+} W^{-}$production [15], where the jets from the two $W$ 's overlap and cross talk, so that the interplay between space-time dynamics and the color flow of close-by partons may lead to a noticeable shift in the experimental $W$ mass determination [16].

Summarizing the above arguments, our motivation in the following is twofold.

(i) First, we would like to provide an alternative and supplementary analysis of standard nondiffractive DIS events, in order to estimate the "background" 1 to the less well understood diffractive, "large-rapidity-gap" events. To the extent that other parton-shower models [17-22] generally describe this background well, our approach should give the same answer, because our additional space-time information which is not contained in previous investigations should not contradict the well-known parton evolution in momentum space. On the other hand, the space-time geography of nondiffractive events may shed some light on the dynamics of diffractive events, which presumably undergo a different space-time development.

(ii) Second, since our approach is, in principle, designed to be universally applicable to high-energy collisions involving lepton, hadron, or nuclear beams, we also see $e p$ collisions at HERA as a learning ground for future $e A$ (HERA?), $p p, p A, A A$ (RHIC, LHC) experiments, whose theoretical description certainly requires knowledge of space-time evolution in order to resolve the complex multi-particle dynamics over the expected long collision time scales.

The paper is organized as follows. In Sec. II we review specific features of DIS at HERA, with the primary aim of establishing our nomenclature and notation for kinematic variables. In Sec. III we introduce the general concept of our model for the space-time development of the hadronic system, recalling relevant aspects of our framework of quantum multiparticle kinetics, the treatment of the initial state, the space-time development of the spacelike and timelike parton showers associated with initial- and final-state radiation, and our spatial criterion for the formation of hadronic clusters and their subsequent decays. Section IV presents our main results, including the time development of the rapidity distribution, inclusive hadronic spectra and transverse energy flow. Particular attention is paid to the distribution of the mass $M_{X}$ of the observed hadronic final state in events without large rapidity gaps, which reflects the details of our cluster formation mechanism and hadronization procedure.

\section{SPECIFIC FEATURES OF DIS AT HERA}

For the purpose of clarity and to define quantities used subsequently, we briefly review in this section some basic notions and kinematics, focusing on the conditions of the $e p$ collider HERA, where an electron beam and a proton beam with four-momenta $p_{e, p} \equiv\left(E, p_{z}, \overrightarrow{0}_{\perp}\right)_{e, p}$ and

$$
E_{e}=27 \mathrm{GeV}, \quad E_{p}=820 \mathrm{GeV}, \sqrt{s}=296 \mathrm{GeV}
$$

collide head-on. For comparison, in the center of mass of electron and proton, the energies are $E_{e} \simeq E_{p}=148 \mathrm{GeV}$, corresponding to a global shift of the proton rapidity as compared to Eq. (1) from $\left|y_{p}\right|=7.46$ to $\left|y_{p}\right|=5.75$.

\section{A. Event types}

The physics at HERA may be separated in two classes of event types, illustrated in Fig. 1, whose definitions are as follows.

\footnotetext{
${ }^{1}$ The term "background" is not to be understood literally, because the contribution of diffractive events with a large rapidity gap at HERA is of the order of $10 \%$, which is still comparably small, although experimentally significant [5].
} 
(a)

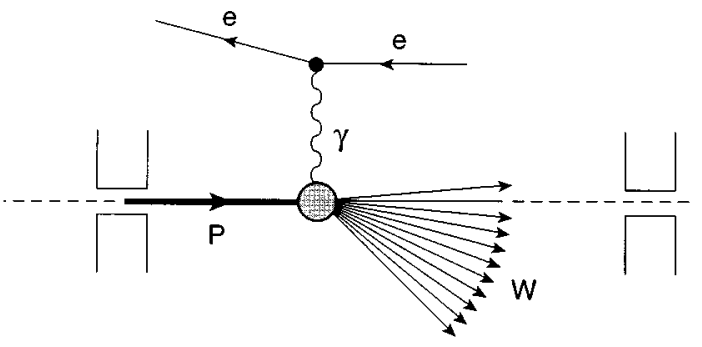

(b)

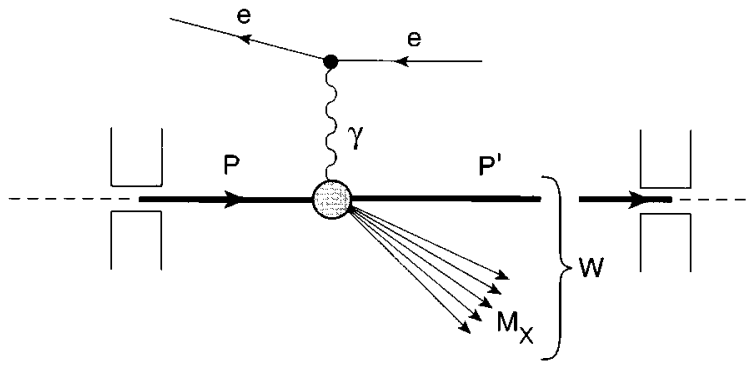

FIG. 1. Schematic diagram of particle production in (a) nondiffractive, and (b) diffractive DIS events. Here, $W$ is the total invariant mass of the produced hadronic system, $M_{X}$ is the mass of the observed final state in the detector, and in (b) $P^{\prime}$ represents the outgoing (excited) proton or low-mass nucleonic resonant state.

(i) Nondiffractive events [Fig. 1(a)]: Here, the exchanged virtual photon ${ }^{2}$ destroys the coherence of the incoming proton by a hard scattering off a quark inside the proton, and breaks up the proton into colored subsystems, which are essentially a jet (led by the struck quark) and the proton remnant system (consisting of the partons that have not taken part in the hard interaction). This class of events is well described by the standard QCD hard scattering picture.

(ii) Diffractive events [Fig. 1(b)]: This class is characterized by an interaction in which the proton either remains intact or receives some small internal excitation to become a relatively low-mass system, and in which the virtual photon also fragments into a relatively low-mass system of particles. This leads generally to experimentally observable large rapidity gaps ${ }^{3}$ between the outgoing proton and the rest of the produced hadronic system, which may be interpreted as the exchange of a colorless object (the "pomeron") between the photon and the proton.

For the remainder of this paper we consider exclusively the nondiffractive event-type, which is describable from first principles in terms of the perturbative QCD parton picture, and for which our space-time approach in terms of photonquark hard scattering, parton shower evolution, and partonhadron conversion, is applicable as an extension of our previous work on $e^{+} e^{-}$collisions $[16,23]$. The diffractive event-type will not be addressed here, since it requires specific model extensions which we want to avoid at this point.

\footnotetext{
${ }^{2}$ In the kinematic region investigated, contributions from $Z^{0}$ exchange can be neglected.

${ }^{3}$ The terms "diffractive events" and "large rapidity-gap events" are often used synonymously.
}

(a)

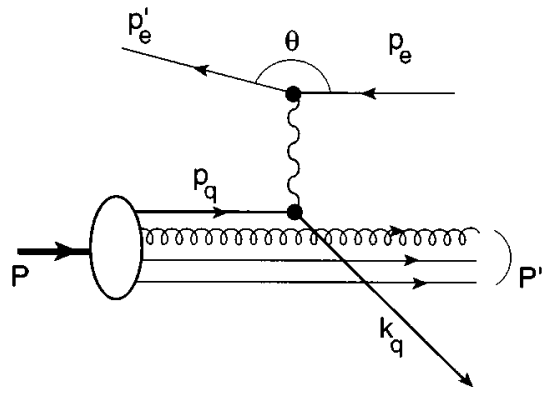

(b)

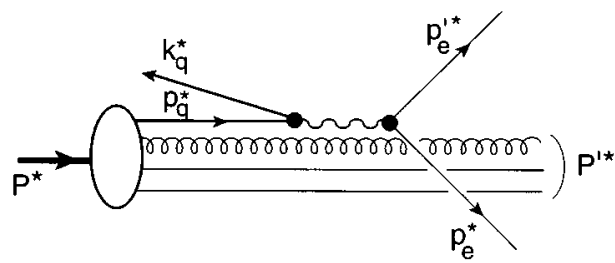

FIG. 2. Deep-inelastic ep scattering viewed in (a) the HERA laboratory frame where $e$ and $p$ collide head-on, and (b) the $\gamma p$ c.m.s., where $\gamma$ and $p$ collide head-on.

\section{B. Kinematics}

The pecularities of the kinematics of DIS in general, and of the HERA facility in particular, require a clear specification of which Lorentz frame is chosen, an issue which is especially important when dealing with the space-time dynamics. The HERA laboratory frame ( $\equiv e p$ lab) is the actual experimental setup [cf. Fig. 2(a)], in which electron and proton beams collide head-on, but with beam momenta that differ by more than an order of magnitude. This is different from the $e p$ center-of-mass frame ( $\equiv e p$ c.m.s.) in which electron and proton have equal but opposite momentum, and which is shifted in rapidity as compared to the laboratory frame. Most convenient for theoretical analyses, however, is the $\gamma p$ center-of-mass frame ( $\equiv \gamma p$ c.m.s. $)$, in which the virtual photon and proton collide head-on [cf. Fig. 2(b)].

Our convention in the following is that frame-dependent quantities generally refer to the $e p$ lab or the $e p$ c.m.s. (which, as mentioned below Eq. (1), is related to the former by a trivial shift of the proton rapidity by 1.7 units), whereas Lorentz noninvariant quantities which refer to the $\gamma p$ c.m.s. are marked by an asterisk. For instance, $E$ and $k_{\perp}$ represent a particle's energy and the momentum transverse to the electron-proton axis in the $e p$ system, respectively, while $E^{*}$ denotes the energy in the $\gamma p$ c.m.s. and $k_{\perp}^{*}$ the momentum transverse to the photon-proton axis. In either frame, we define the negative $z$ axis by the proton direction.

Let $p_{e}(P)$ denote the electron (proton) incoming momenta and $q$ the spacelike photon four-momentum, and define the standard Lorentz invariants for DIS as

$$
s \equiv\left(p_{e}+P\right)^{2}, \quad Q^{2} \equiv-q^{2}, \quad x \equiv \frac{Q^{2}}{2 P \cdot q}, \quad y \equiv \frac{P \cdot q}{P \cdot p_{e}},
$$


TABLE I. Examples of the kinematic relations (2) and (3) between the Bjorken scaling variable $x$, the absolute squared invariant mass of the photon $Q^{2}$, and the inelasticity variable $y$, as well as the total invariant mass $W$ of the hadronic system.

\begin{tabular}{lcccc}
\hline \hline & $Q^{2}$ & "Small" $x$ & "Large" $x$ \\
& $\left(\mathrm{GeV}^{2}\right)$ & $2.33 \times 10^{-4}$ & $1.72 \times 10^{-3}$ & $5 \times 10^{-2}$ \\
\hline & 4 & 0.20 & 0.027 & 0.0009 \\
$y$ & 8 & 0.39 & 0.053 & 0.0018 \\
& 14 & 0.69 & 0.093 & 0.0032 \\
& 28 & 1 & 0.18 & 0.0064 \\
& 54 & 1 & 0.36 & 0.012 \\
& 110 & 1 & 0.73 & 0.025 \\
$W$ & 4 & 48 & 9 \\
$(\mathrm{GeV})$ & 8 & 68 & 12 \\
& 14 & 245 & 127 & 16 \\
& 28 & 296 & 177 & 23 \\
& 54 & 296 & 253 & 32 \\
& 110 & 296 & & 46 \\
\hline \hline
\end{tabular}

in terms of these measured momenta, where $s$ is the total invariant mass squared of the ep system (1), $Q^{2}$ specifies the invariant mass of the photon, and $x, y$ are the usual dimensionless Bjorken variables, commonly termed the "scaling variable" and the "inelasticity parameter," respectively. From these definitions, one finds for $Q^{2} \gg M_{p}^{2}$ that

$$
Q^{2} \approx x y s, \quad W^{2} \equiv(P+q)^{2} \approx Q^{2} \frac{1-x}{x},
$$

where $W$ is the invariant mass of the hadronic system, which equals the total c.m. energy in the $\gamma p$ c.m.s. Table I familiarizes the kinematic relations among variables $x, Q^{2}, y$, and $W^{2}$ with some numerical examples. Figure 3 presents schematically the phase-space regime spanned by these variables, and emphasizes the region in the $x-Q^{2}$ plane which is experimentally investigated at HERA. The region of former fixedtarget experiments is also indicated, corresponding to $y<0.01$ and $Q^{2} \lesssim 100 \mathrm{GeV}^{2}$.

For the purpose of relating the kinematic conditions in the ep lab to the experimental observables measured or calculated in the $\gamma p$ c.m.s., we need the Lorentz transformation of the particle four-vectors $p_{\mu}$ and $p_{\mu}^{*}$. For instance, the fourmomenta of the incoming proton and photon, and of the incoming and outgoing (struck) quark, respectively, are in the ep lab [Fig. 2(a)] given by

$$
\begin{gathered}
P=\left(E_{p}, 0,0,-E_{p}\right) \\
q=\left(y E_{e}-\frac{Q^{2}}{4 E_{e}},-\sqrt{(1-y) Q^{2}}, 0,-y E_{e}-\frac{Q^{2}}{4 E_{e}}\right) \\
p_{q}=\left(x E_{p}, 0,0, x E_{p}\right) \\
k_{q}=\left(y E_{e}+\frac{(1-y) Q^{2}}{4 y E_{e}},-\sqrt{(1-y) Q^{2}}, 0,-y E_{e}\right. \\
\left.+\frac{(1-y) Q^{2}}{4 y E_{e}}\right)
\end{gathered}
$$

where $Q \gg M_{p}$ is assumed. On the other hand, in the preferable $\gamma p$ c.m.s. [Fig. 2(b)], the corresponding momenta are

$$
\begin{gathered}
P^{*}=\frac{2 y E_{e} E_{p}}{\sqrt{4 y E_{e} E_{p}-Q^{2}}}(1,0,0,1), \\
q^{*}=\frac{2 y E_{e} E_{p}}{\sqrt{4 y E_{e} E_{p}-Q^{2}}}\left(1-\frac{Q^{2}}{2 y E_{e} E_{p}}, 0,0,-1\right), \\
p_{q}^{*}=x P^{*}, \\
k_{q}^{*}=\frac{2 y E_{e} E_{p}}{\sqrt{4 y E_{e} E_{p}-Q^{2}}}\left(1-\frac{Q^{2}}{4 y E_{e} E_{p}}, 0,0,-1+\frac{Q^{2}}{4 y E_{e} E_{p}}\right) .
\end{gathered}
$$

The invariant differential cross section for nondiffractive events with a hard photon scattering is the convolution of the elementary photon-quark cross section with the quark and antiquark densities in the struck proton,

$$
\frac{d \sigma}{d x d y}=\sum_{i} \frac{d \hat{\sigma}_{i}}{d x d y} e_{i}^{2} x f_{i}\left(x, Q^{2}\right)
$$

where the index $i$ labels the quark and antiquark flavors, with $e_{i}$ and $f_{i}\left(x, Q^{2}\right)$ denoting the corresponding electric charges and (anti)quark distributions of the proton. The associated elementary cross sections $d \hat{\sigma}_{i}$ are given to lowest order (i.e., before any QCD radiation) by

$$
\begin{aligned}
\frac{d \hat{\sigma}_{i}}{d x d y}= & \frac{2 \pi \alpha_{\mathrm{em}}^{2} e_{i}^{2}}{y Q^{2}}\left\{\left(1+(1-y)^{2}\right)+\frac{4 p_{\perp \text { prim }}^{2}}{Q^{2}}(1-y)\right. \\
& -\frac{4 p_{\perp \text { prim }}}{Q}(2-y) \cos (\phi)+\frac{4 p_{\perp \text { prim }}^{2}}{Q^{2}} \\
& \times(1-y) \cos (2 \phi)\},
\end{aligned}
$$




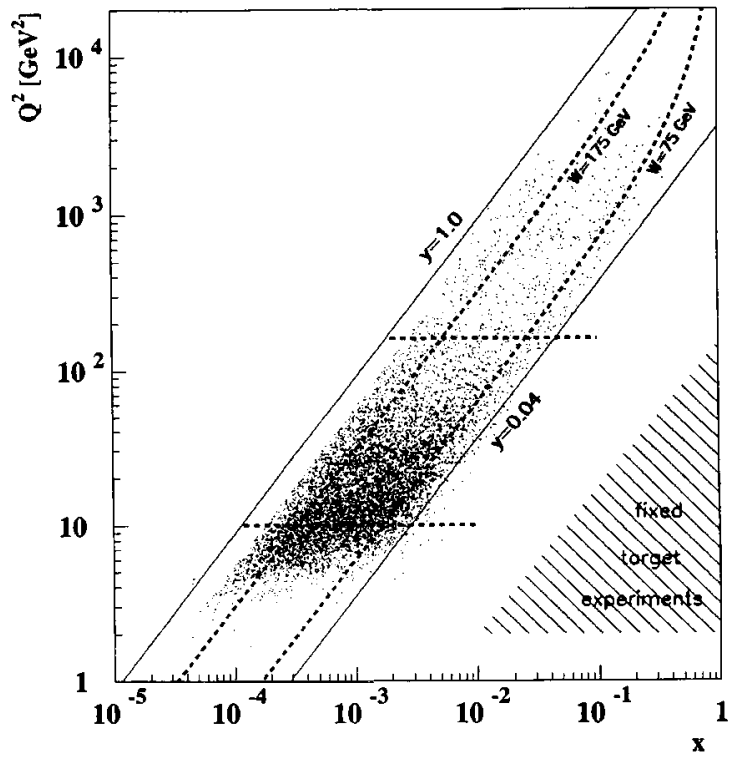

FIG. 3. Contour plot in the $x-Q^{2}$ plane of the mass of the produced hadronic system $W$ and the inelasticity variable $y$ for $\sqrt{s}=296 \mathrm{GeV}$ at HERA. The approximate region of previous fixedtarget experiments is indicated by the shaded area at $x \geqslant 10^{-2}$. (From Ref. [24].)

where $\vec{p}_{\perp \text { prim }}=p_{\perp \text { prim }}(\cos \phi$, $\sin \phi)$ is the intrinsic transverse momentum of the primary, initial quark or antiquark due to the Fermi motion of the partons inside the proton. Intuitively, one expects the value of $p_{\perp \text { prim }}$ to be of the order of the inverse proton radius, and it is, in fact, determined experimentally in hadronic collisions as well as in DIS to be $\approx$ 400-450 MeV [25].

\section{THE MODEL}

\section{A. General concept}

The central element in our approach is the use of QCD transport theory [26] and quantum field kinetics [23] to follow the evolution of a generally mixed multiparticle system of partons and hadrons in seven-dimensional phase space $d^{3} r d^{3} k d k^{0}$. We include both the perturbative QCD partoncascade development [26-29], and the phenomenological parton-hadron conversion model which we have proposed previously in Refs. $[14,16]$, in which we consider dynamical parton-cluster formation as a local, statistical process that depends on the spatial separation and color of nearestneighbor partons, followed by the decay of clusters into hadrons. In contrast with the commonly used momentum-space description, in our approach we trace the microscopic history of the dynamically evolving particle system in space-time and momentum space, so that the correlations of partons in space, time, and color can be taken into account for both the perturbative cascade evolution and the nonperturbative hadronization. We emphasize that one strength of this approach lies in the possible extension of its applicability to the collision dynamics of complicated multiparticle systems, as in $e A, p A$, and $A A$ collisions, for which a causal time evolution in position space and momentum space is essential.

The model contains three main building blocks which generically embody high-energy collisions involving leptons, hadrons, or nuclei in colliders (for DIS ep collisions, the model components are illustrated in Fig. 4): (a) the initial state associated with the incoming collision partners (the beam particles), in particular the phenomenological construction of the hadron (nucleus) in terms of quark and gluon phase-space distributions; (b) the parton cascade development with mutual- and self-interactions of the system of quarks and gluons consisting of both the materialized partons from parton showers, and the spectator partons belonging to the remnants of the collided beam particles; (c) the hadronization of the evolving system in terms of parton coalescence to color-neutral clusters as a local, statistical process that depends on the spatial separation and color of nearestneighbor partons, followed by the decay of clusters into hadrons according to the density of final hadron states.

Such a pragmatical division, which assumes complex interference between the different physics regimes to be negligible, is possible if the respective dynamical scales are such that the short-range hard interaction, with its associated perturbative parton evolution, and the nonperturbative mechanism of hadron formation occur on well-separated spacetime scales. For DIS, this condition of validity requires $\min \left(W^{2}, Q^{2}\right) \geqslant L_{c}^{-2} \gg \Lambda_{\mathrm{QCD}}^{2}$, meaning that the characteristic mass scale for the $\gamma p$ hard scattering and parton shower development ( $W^{2}, Q^{2}$, or a combination of the two) is larger than the inverse "confinement length scale" $L_{c} \sim 1 \mathrm{fm}$ separating perturbative and nonperturbative domains. Specifically, for DIS, it is apparent from Eq. (3) that in the small$x$ regime probed at HERA $\left(10^{-4} \leqq x \leqq 10^{-3}\right)$, one has $60 \lesssim W \leqslant 300 \mathrm{GeV}$ for $10 \leqslant Q^{2} \leqslant 300 \mathrm{GeV}^{2}$, so that the above requirement is well satisfied. We emphasize, however, that in our model the interplay between perturbative and nonperturbative regimes is controlled locally by the space-time evolution of the mixed parton-hadron system itself, rather than by an arbitrary global division between parton and hadron degrees of freedom.

We now turn to the specific case of DIS, and in the following subsections we will discuss the above components in more detail.

\section{B. Framework of quantum kinetics for multiparticle dynamics}

From quantum kinetic theory, one can obtain a space-time description of multiparticle systems in high-energy QCD processes, as has been discussed formally in Ref. [23]. Applied to the concept of our model, as outlined in Sec. III A, this framework allows us to express the time evolution of the mixed system of incoherent partons, composite clusters, and physical hadrons in terms of a closed set of integrodifferential equations for the local phase-space densities of the different particle excitations. The definition of these phase-space densities ("Wigner densities"), denoted by $F_{\alpha}$, where $\alpha \equiv p, c, h$ labels the species of partons, prehadronic clusters, or hadrons, respectively, is

$$
F_{\alpha}(r, k) \equiv F_{\alpha}(t, \vec{r} ; E, \vec{k})=\frac{d N_{\alpha}(t)}{d^{3} r d^{3} k d E},
$$

where $k^{2}=E^{2}-\vec{k}^{2}$ can be off or on mass shell. The densities (8) measure the number of particles of type $\alpha$ at time $t$ with position in $\vec{r}+d \vec{r}$, momentum in $\vec{k}+d \vec{k}$, and energy in 


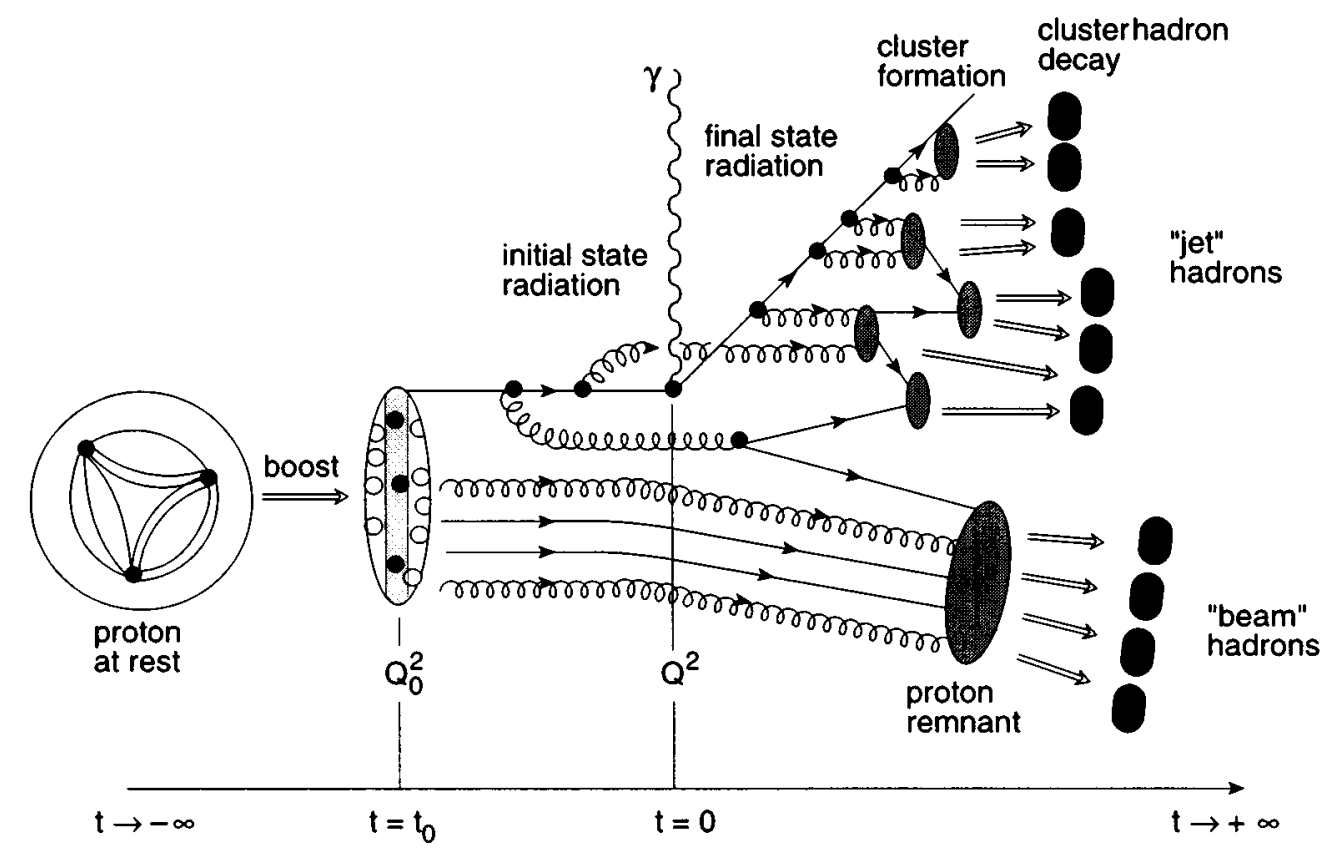

FIG. 4. Schematics of the components of our model for DIS: The highly Lorentz-contracted incoming proton with its initial-state parton configuration evolves from the remote past $t=t<0$, and is struck by the photon at $t=0$. This hard interaction picks a quark out of the proton's parton cloud, thereby triggering initial-state (spacelike) and final-state (timelike) parton showers. With increasing time $t \rightarrow+\infty$, the partons evolve by further radiation, whereas the remnant proton propagates on as a coherent remainder. In the process of hadronization the produced partons may coalesce to colorless clusters if they are nearest neighbors in space-time, whereas the virtual partons of the proton remnant combine with a color-neutralizing parton to form a massive beam cluster. Both "parton clusters" and the "beam clusters," subsequently, convert into primary hadrons that subsequently decay to low-mass final-state particles.

$E+d E$ (or equivalently, invariant mass in $k^{2}+d k^{2}$ ). The $F_{\alpha}$ are the quantum analogues of the classical phase-space distributions, and contain the essential microscopic information required for a statistical description of the time evolution of a many-particle system in complete phase space, thereby providing the basis for calculating macroscopic observables in the framework of relativistic kinetic theory.

The Wigner densities (8) are determined by the selfconsistent solutions of a set of transport equations (in spacetime) coupled with renormalization-group-type equations (in momentum space). Referring to Refs. $[16,14,23]$ for details, we remark that these equations can be generically expressed as convolutions of the densities of radiating or interacting particles $F_{\beta}$ with specific cross sections $\hat{I}_{j}$ for the processes $j$, yielding the following closed set of balance equations for the space-time development of the densities of partons $F_{p}$, clusters $F_{c}$, and hadrons $F_{h}$,

$$
\begin{aligned}
& k \cdot \partial_{r} F_{p}(r, k)= F_{p^{\prime}} \circ \hat{I}\left(p^{\prime} \rightarrow p p^{\prime \prime}\right)-F_{p} \circ \hat{I}\left(p \rightarrow p^{\prime} p^{\prime \prime}\right) \\
&-F_{p} F_{p^{\prime}} \circ \hat{I}\left(p p^{\prime} \rightarrow c\right), \\
& k \cdot \partial_{r} F_{c}(r, k)= F_{p} F_{p^{\prime}} \circ \hat{I}\left(p p^{\prime} \rightarrow c\right)-F_{c} \circ \hat{I}(c \rightarrow h), \\
& k \cdot \partial_{r} F_{h}(r, k)=F_{c} \circ \hat{I}(c \rightarrow h),
\end{aligned}
$$

where $k \cdot \partial_{r} \equiv k_{\mu} \partial / \partial r^{\mu}$. We remark that Eq. (9) implicitly embodies the momentum-space $\left(k^{2}\right)$ evolution of partons through the renormalization of the phase-space densities $F_{p}$, determined by their change $k^{2} \partial F_{p}(r, k) / \partial k^{2}$ with respect to a variation of the mass (virtuality) scale $k^{2}$ in the usual QCD evolution framework [27,28]. ${ }^{4}$ Each of the terms on the right-hand side of Eqs. (9) -(11) corresponds to one of the following categories (cf. Fig. 4): (i) parton multiplication through radiative emission processes on the perturbative level, (ii) colorless cluster formation through parton recombination depending on the local color and spatial configuration, and (iii) hadron formation through decays of the cluster excitations into final-state hadrons. Each convolution $F \circ \hat{I}$ of the density of particles $F$ entering a particular vertex $\hat{I}$ includes a sum over contributing subprocesses, and a phasespace integration weighted with the associated subprocess probability distribution of the squared amplitude.

The Eqs. (9)-(11) reflect a probabilistic interpretation of QCD evolution in space-time and momentum space in terms of sequentially ordered interaction processes $j$, in which the rate of change of the particle distributions $F_{\alpha}(\alpha=p, c, h)$ in a phase-space element $d^{3} r d^{4} k$ is governed by the balance of gain $(+)$ and loss $(-)$ terms. The left-hand side describes free propagation of a quantum of species $\alpha$, whereas on the right-hand side the interaction kernels $\hat{I}$ are integral operators that incorporate the effects of the particles' self and mutual interactions. This quasiclassical, probabilistic character of high-energy particles is essentially an effect of time dilation, because in any frame where the particles move close to the

\footnotetext{
${ }^{4}$ For prehadronic clusters and hadrons, we assume renormalization effects to be comparatively small, so that their mass fluctuations $\Delta k^{2} / k^{2}$ can be ignored to first approximation, implying $k^{2} \partial F_{c}(r, k) / \partial k^{2}=k^{2} \partial F_{h}(r, k) / \partial k^{2}=0$.
} 
speed of light, the associated wave packets are highly localized to short space-time extent, so that long-distance quantum interference effects are generally very small.

\section{Scheme of solution and choice of Lorentz frame}

In the above kinetic approximation [23] to the multiparticle dynamics, the probabilistic character of the evolution Eqs. (9)-(11) allows one to solve for the Wigner densities $F_{\alpha}(r, k)$ by simulating the dynamical development as a Markovian process causally in time. Because it is an initial-value problem, one must specify some physically appropriate initial condition $F_{\alpha}\left(t_{0}, \vec{r}, k\right)$ at starting time $t_{0}$, such that all the dynamics prior to this point is effectively embodied in this initial form of $F_{\alpha}$. The set of kinetic equations (9)-(11) can then be solved in terms of the evolution of the Wigner densities $F_{\alpha}$ for $t>t_{0}$ using Monte Carlo methods to simulate the time development of the mixed system of partons, clusters, and hadrons in position and momentum space $[16,26]$.

In the next subsections we explain in more detail the different components for the case of DIS, namely, the initialstate ansatz, parton-shower development, and parton-hadron conversion. The overall concept of the simulation is illustrated in Fig. 4 and can be summarized as follows: given the initial state of the photon and the proton disassembled into its parton content, the hard interaction of the photon with one of the quarks occurs at time $t=0$. Specifying the initial state at some earlier time $t_{0}<0$, and with the hard scattering variables chosen from the cross section, the phase-space distribution of particles at $t=0$ can be calculated and then evolved in small time steps forward, until stable final-state hadrons are left as freely streaming particles. The size of time steps is chosen as $\Delta t \sim 10^{-3} \mathrm{fm}$, so that an optimal resolution of the particle dynamics in space and energy momentum is achieved. The partons propagate along classical trajectories until they interact, i.e., decay (branching process) or recombine (cluster formation). Similarly, the clusters so formed travel along classical paths until they convert into hadrons (cluster decay). The corresponding probabilities and time scales of interactions are sampled stochastically from the relevant probability distributions in the kernels $\hat{I}$ of Eqs. (9)(11).

It is clear that the description of particle evolution is Lorentz-frame dependent, and a suitable reference frame must be chosen (not necessarily the laboratory frame). When computing Lorentz-invariant quantities, such as cross sections or final-state hadron spectra, the particular choice is irrelevant, whereas for noninvariant observables, such as energy distibutions or space-time-dependent quantities, one must at the end transform from the arbitrarily chosen frame of theoretical description to the actual frame of measurement. Furthermore, at HERA even experimental analyses are often carried out in the $\gamma p$ c.m.s. (5), rather than the $e p$ lab (4). For our purposes, it is most convenient to choose the overall center-of-mass frame of the colliding electron and proton, the $e p$ c.m.s., as the global frame with respect to which the evolution of the collision system is followed. ${ }^{5} \mathrm{Re}-$

\footnotetext{
${ }^{5}$ However, to make contact with the HERA experiments, most of our results will be discussed later in the $\gamma p$ c.m.s. rather than the ep c.m.s., unless specified otherwise.
}

call our convention that the ep collision axis defines the $z$ axis, with the electron (proton) moving in the positive (negative) $z$ direction. The incoming four-momenta $p_{e}$ and $P$ involve, therefore, no transverse components, and are ${ }^{6}$

$$
\begin{gathered}
p_{e}=\left(\frac{s+m_{e}^{2}-M_{p}^{2}}{2 \sqrt{s}}, 0,0,+P_{\text {c.m. }}\right) \approx \frac{\sqrt{s}}{2}(1,0,0,1), \\
P=\left(\frac{s-m_{e}^{2}+M_{p}^{2}}{2 \sqrt{s}}, 0,0,-P_{\text {c.m. }}\right) \approx \frac{\sqrt{s}}{2}(1,0,0,-1),
\end{gathered}
$$

where $P_{\text {c.m. }}=\sqrt{s-\left(m_{e}+M_{p}\right)^{2}} \sqrt{s-\left(m_{e}-M_{p}\right)^{2}} /(2 \sqrt{s})$ is the ep c.m. momentum.

\section{Initial state}

The incoming electron is considered as a pointlike object carrying the full beam energy, meaning that we neglect any QED or QCD substructure of the electron, as well as initialstate photon radiation by the electron. We assume that the electron emits the virtual photon of invariant mass $Q^{2}=-q^{2}$ at time $t=-Q^{-1}$, so that $t=0$ characterizes the point when the photon hits the incoming proton, as is depicted in Fig. 4.

The incoming proton, on the other hand, is decomposed into its parton substructure by phenomenological construction of the momentum and spatial distributions of its daughter partons on the basis of the experimentally measured proton structure functions and elastic proton form factor. Here, it is important to distinguish between the scales $Q^{2}$ and $Q_{0}^{2}$ (cf. Fig. 4): The hard scattering scale $Q^{2}=-q^{2}$ is set by the momentum transfer $q$ between electron and proton and determines the parton structure as seen by the virtual $\gamma$ after the initial-state radiation of the struck quark. The initialresolution scale $Q_{0}^{2}$, on the other hand, determines how detailed the parton phase-space density in the proton would be resolved before the initial-state radiation. Hence, in accord with Eq. (8), we introduce the initial parton phase-space distribution $F_{a}^{(0)}(r, p)$ as the number density of partons in a phase-space element $d^{3} r d^{3} p d E$ at time $t=t_{0}$ within the proton at an initial resolution scale $Q_{0}^{2}=1 \mathrm{GeV}^{2}$. We assume the factorized form

$$
\begin{aligned}
F_{a}^{(0)}(r, p) & \left.\equiv F_{a}(r, p)\right|_{t=t_{0},\left|p^{2}\right| \simeq Q_{0}^{2}} \\
& =\left.P_{a}\left(\vec{p}, \vec{P} ; Q_{0}^{2}\right) \circ R_{a}(\vec{p}, \vec{r}, \vec{R})\right|_{t=t_{0}} .
\end{aligned}
$$

The right-hand side $P_{a}{ }^{\circ} R_{a}$ is a convolution of an initialmomentum distribution $P_{a}$ and a spatial distribution $R_{a}$, with the subscript $a=g, q_{i}, \overline{q_{i}}$ labeling the parton species (gluons or (anti)quarks of flavor $i=1, \ldots, n_{f}$ ). The fourvectors $p \equiv p^{\mu}=(E, \vec{p})$ and $r \equiv r^{\mu}=(t, \vec{r})$ refer to the partons, whereas $\vec{P}=\left(0,0,-P_{\text {c.m. }}\right)$ and $\vec{R} \equiv \overrightarrow{0}$ refer to the initial threemomentum and the position of the parent proton at $t=t_{0}$ in

\footnotetext{
${ }^{6}$ We emphasize that in our calculations we use exact kinematics, and take into account proton, electron, and quark masses.
} 
the $e p$ c.m.s. The partons' energies $E=\sqrt{\vec{p}^{2}-Q_{0}^{2}}$ take into account initial-spacelike virtualities $p^{2}<0$, which reflects the fact that before the collision the partons are confined inside the parent proton and cannot be treated as free particles (meaning that they do not have enough energy to be on mass shell, but are spacelike off shell). The initial momentum distribution is taken as

$$
P_{a}\left(\vec{p}, \vec{P} ; Q_{0}^{2}\right)=\left(\frac{x}{\tilde{x}}\right) f_{a}\left(x, Q_{0}^{2}\right) g\left(\vec{p}_{\perp}\right) \delta\left(P_{z}-\frac{\sqrt{s}}{2}\right) \delta^{2}\left(\vec{P}_{\perp}\right) .
$$

Here, $x$ and $\widetilde{x}$ are the partons' longitudinal momentum and energy fractions, respectively,

$$
x=\frac{p_{z}}{P_{z}}, \quad \tilde{x}=\frac{E}{E_{p}}=\sqrt{x^{2}+\frac{p_{\perp}^{2}-Q_{0}^{2}}{E_{p}^{2}}}
$$

and the functions $f_{a}\left(x, Q_{0}^{2}\right)$ are the usual (measured) quark and gluon structure functions of the proton, ${ }^{7}$ which specify the longitudinal momentum distribution, whereas the transverse momentum distribution $g\left(\vec{p}_{\perp}\right)=$ $\left(2 \pi p_{0}^{2}\right)^{-1} \exp \left[-\vec{p}_{\perp}^{2} / p_{0}^{2}\right]$ takes into account the uncertainty of the transverse momentum ("Fermi motion") due to the fact that the initial partons are confined within the proton. The latter is inferred from experimental analyses [25], with $p_{0}=0.42 \mathrm{GeV}$, corresponding to the mean primordial transverse parton momentum $\left\langle\left|\vec{p}_{\perp}\right|\right\rangle$. The normalization is such that

$$
\begin{gathered}
\sum_{a} \int_{0}^{1} d x x f_{a}\left(x, Q_{0}^{2}\right)=1, \quad \int_{0}^{\infty} d^{2} p_{\perp} g\left(\vec{p}_{\perp}\right)=1, \\
\sum_{a} \int d p^{2} \frac{d^{3} p}{(2 \pi)^{3}(2 E)} E P_{a}\left(\vec{p}, \vec{P} ; Q_{0}^{2}\right) \equiv n\left(P, Q_{0}^{2}\right),
\end{gathered}
$$

where $n\left(P, Q_{0}^{2}\right)$ has dimension $1 /$ volume and gives the total number density of partons in the proton with momentum $P$, when resolved at the scale $Q_{0}^{2}$. Finally, we impose the constraint that the total invariant mass of the partons equals the proton mass $M_{p}$ :

$$
\left(\sum_{j} E_{j}\right)^{2}-\left(\sum_{j} p_{x_{j}}\right)-\left(\sum_{j} p_{y_{j}}\right)^{2}-\left(\sum_{j} p_{z_{j}}\right)^{2}=M_{p}^{2}
$$

where the summation $j=1, \ldots, n\left(P, Q^{2}\right)$ runs over all partons resolved at $Q^{2}$, as constrained by Eqs. (16) and (17). With the partons' three-momenta determined from the distributions in $x$ and $\vec{p}_{\perp}$, the requirement (18) fixes the relation between energy and momentum by assigning to each parton an initial-spacelike virtuality such that $p^{2}=E^{2}-\vec{p}^{2}<0$. With this prescription, the resulting distribution in $p^{2}$ is approxi-

\footnotetext{
${ }^{7}$ We use the Glück-Reya-Vogt (GRV) structure function parametrization [29], which describes quite accurately the HERA data even at low $Q^{2}$ and very small $x$.
}

mately Gaussian with a mean value of $\sqrt{\left\langle p^{2}\right\rangle} \approx 500 \mathrm{MeV}$, i.e., the typical initial virtuality of the partons is about $Q_{0} / 2$.

\section{E. Parton-cascade development}

With the above construction of the initial state in terms of the incoming electron and photon, and the parton cloud of the proton, the dynamical development of the system can now be traced according to the kinetic Eqs. (9)-(11), starting from $t=0$. In our statistical picture, the initial-state parton ensemble represents a particular fluctuation of the proton wave function that has developed between $t=t_{0} \simeq Q_{0}^{-1}<0$ and time $t=0$, at which the photon with resolution $Q^{2}$ picks according to the cross section (6), a quark with specific flavor and momentum $p=x P$ out of the incoming parton cloud, while the other partons are viewed as unaffected by the short-range $\gamma q$ interaction. Consequently, as illustrated in Fig. 4, the early stage of the time evolution is characterized by two different physics elements: (a) the parton showers initiated by the quark that is struck out of the original proton wave function through the momentum transfer from the virtual photon, and (b) the propagation of the remnant system consisting of the other initial partons, that remain spectators of the hard process and form the coherent remnant of the original proton.

For the parton shower development we employ the wellestablished jet calculus [30,31] based on the "modified leading logarithmic approximation", (MLLA) to the QCD evolution of hard processes $[27,28]$. A parton shower then reduces to a strictly ordered sequence of elementary branchings $q \rightarrow q g, g \rightarrow g g, g \rightarrow q \bar{q}$, which can be described stochastically as a Markov cascade in position and momentum space. We distinguish initial-state, spacelike branchings of the selected quark before it reaches the $\gamma q$ vertex, and final-state, timelike radiation off the struck quark after the hard $\gamma q$ interaction $[22,32] .{ }^{8}$ The separation into two "hemispheres" divided by the $\gamma q$ vertex is illustated in Fig. 5: it refers to both the chronological order along the real time axis and to the order of emission vertices in momentum space. The initial quark that is picked out by the photon evolves from the remote past $t=t_{0}<0$ towards the hard interaction by sequential branchings $p_{j} \rightarrow p_{j+1}+p_{j+1}^{\prime}$, in each of which one of the daughters continues with increasing spacelike virtuality $\left|p_{j+1}^{2}\right|>\left|p_{j}^{2}\right|$ (where $p_{j}^{2}, p_{j+1}^{2}<0$ ), while the other one acquires a timelike virtuality $p_{j+1}^{\prime 2}>0$ and may develop a timelike shower of its own. The spacelike shower is consequently characterized by increasing virtualities $\left|p_{j}^{2}\right|<\left|p_{j+1}^{2}\right|$, decreasing energies, and increasing opening angles, as the quark approaches the hard vertex at $t=0$ with $\left|p_{n}^{2}\right| \approx Q^{2}$. Once the evolved quark has been struck by the photon, the momentum transfer provides the outgoing quark with enough energy momentum to become a real excitation at $t=0$ and to obtain a timelike virtuality $k_{m}^{2} \approx Q^{2}$. This materialized quark initiates now a shower of sequential timelike branchings

\footnotetext{
${ }^{8}$ This separation implies the neglect of interference between the initial- and final-state showers, a common conceptual defect that is approximately cured by matching on to the lowest-order $O\left(\alpha_{s}\right)$ matrix element.
} 


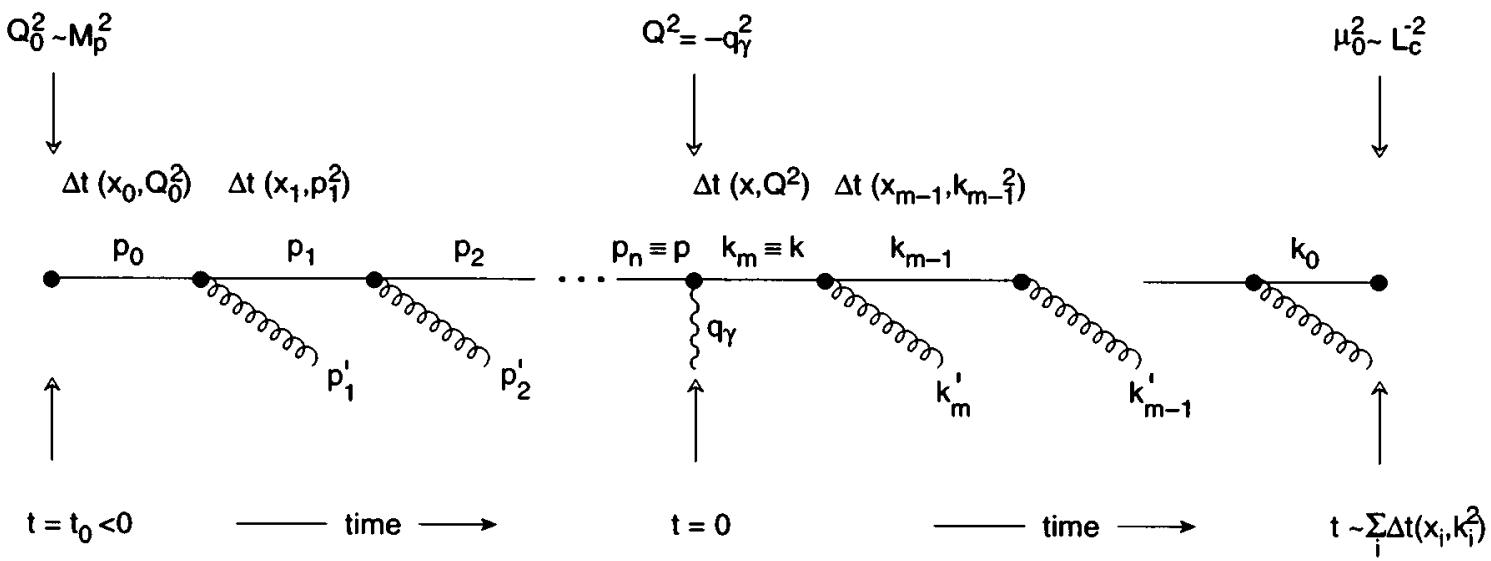

FIG. 5. Schematic diagram of the probabilistic parton evolution in the MLLA framework (solid lines are quarks, curly lines are gluons). The initial-state quark with spacelike virtuality $-p_{0}^{2} \approx Q_{0}^{2}$ evolves from $t=t_{0}$ forward in time and toward the hard $\gamma q$ vertex by successively increasing its off-shellness up to $-p_{n}^{2} \approx Q^{2}$, when it is struck by the photon at $t=0$. The outgoing quark is provided by the momentum transfer with a timelike virtuality $k_{m}^{2} \approx Q^{2}$, and radiates off its excitation by successive gluon radiation until it starts to hadronize by coalescence with another parton, at which point the shower terminates naturally.

$k_{m} \rightarrow k_{m-1}+k_{m-1}^{\prime}$ in which both daughters are timelike (i.e., $k_{m-1}^{2}, k_{m-1}^{\prime 2}>0$ ) with decreasing virtualities $k_{m-1}^{2}<k_{m}^{2}$ decreasing energies, and decreasing opening angles. The branching chain continues into the remote future until it is terminated by the hadronization, which we model as the coalescence of neighboring partons in a cascade, followed by conversion to hadrons (Sec. III F below).

The specific feature of our approach is that, in addition to the definite virtuality and momentum, each elementary vertex has a certain space and time position which is obtained by assuming that the partons in the shower propagate on straight-line trajectories in between the branchings. In the MLLA framework, the basic properties of both spacelike and timelike showers are determined by the Dokshitzer-GribovLipatov-Altarelli-Parisi (DGLAP) equations [33], but with essential differences in time ordering, kinematics, and the treatment of infrared singularities associated with soft gluon emission.

\section{Spacelike parton shower}

As mentioned above and depicted in Fig. 5, the spacelike cascade starts at some time $t=t_{0} \simeq-Q_{0}^{-1}$ before the actual hard scattering at $t=0$, with the initiating parton of virtuality $\left|p_{0}^{2}\right| \simeq Q_{0}^{2}=M_{p}^{2}$ embodied in the parton cloud of the incoming proton, and proceeds up to $p^{2} \equiv p_{n}^{2} \simeq-Q^{2}$ at the hard vertex set by the spacelike photon virtuality. The emitted partons on the side branches, on the other hand, are not connected directly with the $\gamma q$ vertex, but evolve independently as timelike quanta. In the cascade sequence both collinear and soft coherent branchings are properly included [34], if the development of the chain is described in terms of "angularordering" variables (rather than the virtualities $p_{j}^{2}$ ),

$$
\begin{array}{r}
\widetilde{p}_{j}^{2} \equiv E_{j}^{2} \zeta_{j+1}, \quad \zeta_{j+1}=\frac{p_{0} \cdot p_{j+1}^{\prime}}{E_{0} E_{j+1}^{\prime}} \simeq 1-\cos \theta_{0, j+1} \\
\quad(0 \leqslant j \leqslant n),
\end{array}
$$

where $p_{j}=\left(E_{j}, \vec{p}_{j}\right)$ and $p_{j+1}^{\prime}=\left(E_{j+1}^{\prime}, \vec{p}_{j+1}^{\prime}\right)$ are assigned as in Fig. 5 for the $j$ th branching $p_{j} \rightarrow p_{j+1} p_{j+1}^{\prime}$. The spacelike cascade is then strictly ordered in the variable $\widetilde{p}_{j+1}^{2}>\widetilde{p}_{j}^{2}$ which is equivalent to the ordering of emission angles, $E_{j} \theta_{0, j+1^{\prime}}<E_{j+1} \theta_{0, j+2}$.

Because the presence of the external hard interaction at $t=0$ and $Q^{2}$ sets a physical boundary condition on the kinematical evolution of the cascade, it is technically advantageous to reconstruct the cascade backwards in time starting from $t=0$ at the hard vertex $Q^{2}$ and trace the history of the struck quark back to $Q_{0}^{2}$ at $t=t_{0}$. The method used here is a space-time generalization of the "backward evolution scheme" [32,35]. To sketch the procedure, consider the spacelike branching $p_{n-1} \rightarrow p_{n} p_{n}^{\prime}$ which is closest to the $\gamma q$ vertex in Fig. 5. The virtualities satisfy [31] $\left|p_{n}^{2}\right|>\left|p_{n-1}^{2}\right|$, and $p_{n}^{2}, p_{n-1}^{2}<0$ (spacelike) but $p_{n}^{\prime 2}>0$ (timelike). The relative probability for this branching to occur between $\widetilde{p}^{2}$ and $\widetilde{p}^{2}+d \widetilde{p}^{2}$ is given by

$$
\begin{aligned}
& d \mathcal{P}_{n-1, n}^{(S)}\left(x_{n-1}, x_{n}, \tilde{p}^{2} ; \Delta t\right) \\
& =\frac{d \widetilde{p}^{2}}{\widetilde{p}^{2}} \frac{d z}{z} \frac{\alpha_{s}\left((1-z) \tilde{p}^{2}\right)}{2 \pi} \gamma_{n-1 \rightarrow n n^{\prime}}(z) \\
& \quad \times\left(\frac{F\left(r_{n-1} ; x_{n-1}, \tilde{p}^{2}\right)}{F\left(r_{n} ; x_{n}, \tilde{p}^{2}\right)}\right) \mathcal{T}^{(S)}(\Delta t),
\end{aligned}
$$


where $x_{j}=\left(p_{j}\right)_{z} / P_{z}(j=n, n-1)$ are the fractions of longitudinal proton momentum $P_{z}$, with $F\left(r_{j} ; x_{j}, \tilde{p}^{2}\right) \equiv F\left(r_{j}, p_{j}\right)$ the corresponding parton distriibutions introduced before, and the variables

$$
z=\frac{E_{n}}{E_{n-1}} \simeq \frac{x_{n}}{x_{n-1}}, \quad 1-z=\frac{E_{n}^{\prime}}{E_{n-1}} \simeq \frac{x_{n-1}-x_{n}}{x_{n-1}}
$$

specify the fractional energy or longitudinal momentum of parton $n$ and $n^{\prime}$, respectively, taken away from $n-1$. The function $\alpha_{s} /\left(2 \pi \tilde{p}^{2}\right) \gamma(z)$ is the usual DGLAP branching probability in the MLLA, with $\gamma(z)$ giving the energy distribution in the variable $z$. The last factor in Eq. (20) determines the time interval in the ep c.m.s., $\Delta t=t_{n}-t_{n-1}$, that is associated with the branching process $n-1 \rightarrow n n^{\prime}$. We take here simply

$$
\mathcal{T}^{(S)}(\Delta t)=\delta\left(\frac{x_{n}-x_{n-1}}{\left|p_{n}^{2}\right|} P_{z}-\Delta t\right),
$$

which accounts for the formation time of $n$ by its mother $n-1$ on the basis of the uncertainty principle: $\Delta t=\Delta E /\left|p_{n}^{2}\right|, \Delta E \simeq\left(x_{n}-x_{n-1}\right) P_{z}$.

The "backwards evolution" of the spacelike branching $p_{n-1} \rightarrow p_{n}+p_{n}^{\prime}$ is expressed in terms of the probability that parton $(n-1)$ did not branch between the lower bound $\widetilde{p}_{0}^{2}$, given by the initial resolution scale $Q_{0}^{2}$, and $\widetilde{p}^{2}$. In that case, parton $n$ cannot originate from this branching, but must have been produced otherwise or already been present in the initial parton distributions. This nonbranching probability is given by the Sudakov form factor for spacelike branchings:

$$
\begin{aligned}
S_{n}\left(x_{n}, \widetilde{p}^{2}, \widetilde{p}_{0}^{2} ; \Delta t\right)= & \exp \left\{-\sum_{a} \int_{\widetilde{p}_{0}^{2}}^{\widetilde{p}^{2}} \int_{z_{-}\left(\widetilde{p}^{\prime}\right)}^{z_{+}\left(\widetilde{p}^{\prime}\right)} d \mathcal{P}_{n, n-1}^{(S)}\right. \\
& \left.\times\left(x_{n}, z, \widetilde{p}^{\prime 2} ; \Delta t\right)\right\},
\end{aligned}
$$

where the sum runs over the possible species $a=g, q, \bar{q}$ of parton $n-1$. The upper limit of the $\widetilde{p}^{2}$ integration is set by $\widetilde{p}^{2} \leq Q^{2}$, associated with the scattering vertex of quark $n$ with the photon in Fig. 5. The limits $z_{ \pm}$are determined by kinematics [34]: $z_{-}(\widetilde{p})=Q_{0} / \widetilde{p}$ and $z_{+}(\widetilde{p})=1-Q_{0} / \widetilde{p}$. The knowledge of $S_{n}\left(x_{n}, \widetilde{p}^{2}, Q_{0}^{2}\right)$ is enough to trace the evolution of the branching closest to the hard vertex backwards from $p_{n}^{2}$ at $t=t_{n} \equiv 0$ to $p_{n-1}^{2}$ at $t_{n-1}=-x_{n} /\left|p_{n}^{2}\right| P_{z}$. The next preceding branchings $p_{n-2} \rightarrow p_{n-1} p_{n-1}^{\prime}$, etc., are then reconstructed in exactly the same manner with the replacements $t_{n} \rightarrow t_{n-1}, x_{n} \rightarrow x_{n-1}, p_{n}^{2} \rightarrow p_{n-1}^{2}$ and so forth, until the initial point $p_{0}^{2}$ at $t_{0}=-Q_{0}^{-1}$ is reached.

\section{Timelike parton shower}

Timelike cascades are initiated by the secondary partons, i.e., those that emerge from the side branches of the initialstate radiation from the scattering quark before $t=0$, as well as those that are produced by final-state emission from the scattered quark after $t=0$. Consider the timelike cascade in Fig. 5 that is initiated by the outgoing quark of momentum $k \equiv k_{m}$ emerging from the hard vertex at $t=0$ and off shell by an amount $k^{2} \equiv k_{m}^{2} \leq Q^{2}$.
Again, an angular-ordered (rather than virtuality-ordered) time evolution of the cascade is employed to incorporate interference effects of soft gluons emitted along the tree in Fig. 5. In contrast with (19), the timelike version of the angular evolution variable is [36]

$$
\widetilde{k}_{j}^{2} \equiv E_{j}^{2} \xi_{j-1}, \quad \xi_{j-1}=\frac{p_{j-1} \cdot p_{j-1}^{\prime}}{E_{j-1} E_{j-1}^{\prime}} \simeq 1-\cos \theta_{(j-1),(j-1)^{\prime}}
$$

$$
(m \geqslant j \geqslant 1)
$$

so that the timelike cascade can be described by a $\widetilde{k^{2}}$-ordered (rather than $k^{2}$-ordered) evolution, which corresponds to an angular ordering with decreasing emission angles $\theta_{j, j^{\prime}}>\theta_{(j-1),(j-1)^{\prime}}$.

Proceeding analogously to the spacelike case [cf. Eq. (20)], the probability $d \mathcal{P}_{m, m-1}^{(T)}$ for the first branching after the $\gamma q$ vertex, $k_{m} \rightarrow k_{m-1} k_{m-1}^{\prime}$ with $k_{m-1}^{2}, k_{m-1}^{\prime 2}$, is given by the space-time extension $[23,26]$ of the usual DGLAP probability distribution [33],

$$
\begin{aligned}
d \mathcal{P}_{m, m-1}^{(T)}\left(z, \widetilde{k^{2}} ; \Delta t\right)= & \frac{d \widetilde{k}^{2}}{\widetilde{k}^{2}} d z \frac{\alpha_{s}\left(\kappa^{2}\right)}{2 \pi} \gamma_{m \rightarrow(m-1),(m-1)^{\prime}}(z) \\
& \times \mathcal{T}^{(T)}(\Delta t)
\end{aligned}
$$

where $\mathcal{T}^{(T)}(\Delta t)$ is the probability that parton $m$ with virtuality $k_{m}^{2}$ and corresponding proper lifetime $\tau_{m} \propto 1 / \sqrt{k_{m}^{2}}$ decays within a time interval $\Delta t$ :

$$
\mathcal{T}^{(T)}(\Delta t)=1-\exp \left(-\frac{\Delta t}{t_{m}(k)}\right)
$$

The actual lifetime of the decaying parton $m$ in the $e p$ c.m.s. is then $t_{m}(k)=\gamma / \tau_{m}(k)$, where $t_{q}(k) \approx 3 E /\left(2 \alpha_{s} k^{2}\right)$ for quarks and $t_{g}(k) \approx E /\left(2 \alpha_{s} k^{2}\right)$ for gluons [8]. As before, $F_{j}$ denotes the local density of parton species $j=m, m-1$, and $\alpha_{s} /(2 \pi \xi) \gamma(z)$ is the DGLAP branching kernel with energy distribution $\gamma(z)$. The probability (25) is formulated in terms of the energy fractions carried by the daughter partons,

$$
z=\frac{E_{m-1}}{E_{m}}, \quad 1-z=\frac{E_{m-1}^{\prime}}{E_{m}},
$$

with the virtuality $k_{m}$ of the quark $m$ related to $z$ and $\xi$ through $k_{m}^{2}=k_{m_{1}}^{2}+k_{m-1}^{\prime 2}+2 E_{m}^{2} z(1-z) \xi$, and the argument $\kappa^{2}$ in the running coupling $\alpha_{s}$ in Eq. (25) is [34] $\kappa^{2}=2 z^{2}(1-z)^{2} E_{m}^{2} \xi \simeq k_{\perp}^{2}$.

The branching probability (25) determines the distribution of emitted partons in both coordinate and momentum space, because the knowledge of four-momentum and lifetime (or $\Delta t$ between successive branchings) gives the spatial positions of the partons, if they are assumed to propagate on straight paths between the vertices. The probability that parton $m$ does not branch between $\widetilde{k}^{2}$ and a minimum value $\vec{k}_{0}^{2} \equiv \mu_{0}^{2}$ is given by the exponentiation of Eq. (25), yielding the Sudakov form factor for timelike branchings: 


$$
\begin{aligned}
T_{m}\left(\widetilde{k^{2}}, \widetilde{k_{0}^{2}} ; \Delta t\right)= & \exp \left\{-\int_{\tilde{k}_{0}^{2}}^{\widetilde{k}^{2}} \sum_{a} \int_{z_{-}\left(\tilde{k}^{\prime}\right)}^{z_{+}\left(\tilde{k}^{\prime}\right)} d \mathcal{P}_{m, m-1}^{(T)}\right. \\
& \left.\times\left(z, \widetilde{k^{\prime}} ; \Delta t\right)\right\},
\end{aligned}
$$

which is summed over the species $a=g, q, \bar{q}$ of parton $m-1$. The integration limits $\widetilde{k_{0}^{2}}$ and $z_{ \pm}$are determined by the requirement that the branching must terminate when the partons enter the nonperturbative regime and begin to hadronize. As we discuss later, this condition can be parametrized by the confinement length scale $L_{c} \sim 1 \mathrm{fm}$ with $\widetilde{k}_{0}^{2} \gtrsim L_{c}^{-2} \equiv \mu_{0}^{2}$ and $z_{+}\left(\widetilde{k_{m}}\right)=1-z_{-}\left(\widetilde{k_{m}}\right)=\mu_{0} / \sqrt{4 \widetilde{k}_{m}^{2}}$, so that for $z_{+}\left(\widetilde{k}_{0}^{2}\right)=z_{-}\left(\widetilde{k}_{0}^{2}\right)=1 / 2$ the phase space for the branching vanishes.

The Sudakov form factor (28) determines the fourmomenta and positions of the partons of a particular emission vertex as we sketched above for the first branching, but subsequent branchings are described completely analogously by replacing $t_{m} \rightarrow t_{m-1}, x_{m} \rightarrow x_{m-1}, k_{m}^{2} \rightarrow k_{m-1}^{2}$, etc. Hence, $T\left(\widetilde{k^{2}}, \widetilde{k}_{0}^{2} ; \Delta t\right)$ generates the timelike cascade as sequential branchings starting from $t=0$ at the hard vertex forward in time, until the partons eventually hadronize as discussed below.

\section{F. Cluster formation and hadronization}

Both the cluster formation from the collection of quarks and gluons at the end of the perturbative phase and the subsequent cluster decay into final hadrons consist of two components: (i) the recombination of the secondary timelike partons, their conversion into colorless parton clusters, and the subsequent decay into secondary hadrons; (ii) the recombination of the primary spacelike partons that remained spectators throughout the collision development into beam clusters and the fragmentation of these clusters.

The important assumption here is that the process of hadron formation depends only on the local space-, time-, and color-structure of the parton system, so that the hadronization mechanism can be modeled as the formation of colorsinglet clusters of partons as independent entities (prehadrons), which subsequently decay into hadrons. This concept is reminiscent of the "preconfinement" property [37] of parton evolution, which is the tendency of the produced partons to arrange themselves in color-singlet clusters with limited extension in both position and momentum space, so that it is suggestive to suppose that these clusters are the basic units out of which hadrons form.

\section{Cluster formation}

a. Parton clusters. Parton clusters are formed from secondary partons, i.e., those that have been produced by the hard interaction and the parton-shower development. The coalescence of these secondary partons to color-neutral clusters has been discussed in detail in Refs. $[14,16]$, so that we confine ourselves here to the essential points. Throughout the dynamically-evolving parton-shower development, we consider every parton and its nearest spatial neighbor as a potential candidate for a two-parton cluster, which, if color neutral, plays the role of a "preconfined" excitation in the process of hadronization. Within each single time step, the probability for parton-cluster conversion is determined for each nearest-neighbor pair by the requirement that the total color charge of the two partons must give a composite colorsinglet state, and the condition that their relative spatial distance $L$ exceeds the critical confinement length scale $L_{c}$. We define $L$ as the Lorentz-invariant distance $L_{i j}$ between parton $i$ and its nearest neighbor $j$ :

$$
L\left(r_{i}, r_{j}\right)=L_{i j} \equiv \min \left(\Delta_{i 1}, \ldots, \Delta_{i j}, \ldots, \Delta_{i n}\right),
$$

where $\Delta_{i j} \equiv \sqrt{r_{i j}^{\mu} r_{i j, \mu}}, r_{i j}=r_{i}-r_{j}$, and the probability for the coalescence of the two partons $i, j$ to form a cluster is modeled by a distribution of the form

$$
\begin{array}{r}
\Pi_{i j \rightarrow c} \propto\left[1-\exp \left(-\Delta F L_{i j}\right)\right] \simeq 1-\exp \left(\frac{L_{0}-L_{i j}}{L_{c}-L_{i j}}\right) \\
\text { if } L_{0}<L_{i j} \leqslant L_{c},
\end{array}
$$

and 0 (1) if $L_{i j}<L_{0}\left(L_{i j}>L_{c}\right)$. Here, $\Delta F$ is the local change in the free energy of the system that is associated with the conversion of the partons to clusters, and the second expression on the right side is our parametrization in terms of $L_{0}=0.6 \mathrm{fm}$ and $L_{c}=0.8 \mathrm{fm}$ that define the transition regime. As we studied in Ref. [16], the aforementioned color constraint, that only colorless two-parton configurations may produce a cluster, can be incorporated by allowing coalescence for any pair of color charges, as determined by the space-time separation $L_{i j}$ and the probability (30), however, accompanied by the additional emission of a gluon or quark that carries away any unbalanced net color in the case that the two coalescing partons are not in a colorless configuration.

b. Beam clusters. The remaining fraction of the longitudinal momentum and energy that has not been redirected and harnessed by the interaction with the photon is carried by the primary partons of the initial proton, which remained spectators throughout. In our approach these partons maintain their originally assigned momenta and their spacelike virtualities. Representing the beam remnant, they may be pictured as the coherent relics of the original proton wave function. Therefore, the primary virtual partons must be treated differently than the secondary partons which are real excitations that contribute incoherently to the hadron yield. In the $e p$ c.m.s. the primary partons are grouped together to form a massive beam cluster with its four-momentum given by the sum of the parton momenta and its position given by the three-vector mean of the partons' positions.

\section{Hadronization of clusters}

a. Parton clusters. For the decay of each parton cluster into final-state hadrons, we employ the scheme presented in Refs. [14,16,38]: If a cluster is too light to decay into a pair of hadrons, it is taken to represent the lightest single meson that corresponds to its partonic constituents. Otherwise, the cluster decays isotropically in its rest frame into a pair of hadrons, either mesons or baryons, whose combined quantum numbers correspond to its partonic constituents. The corresponding decay probability is chosen to be 


$$
\Pi_{c \rightarrow h}=\mathcal{T}_{c}\left(E_{c}, m_{c}^{2}\right) \mathcal{N} \int_{m_{h}}^{m_{c} d m} \frac{\overline{m^{3}}}{\exp }\left(-\frac{m}{m_{0}}\right),
$$

where $\mathcal{N}$ is a normalization factor, and the integrand is a Hagedorn spectrum [39] that parametrizes quite well the density of accessible hadronic states below $m_{c}$ which are listed in the particle data tables, and $m_{0}=m_{\pi}$. In analogy to Eq. (26), $\mathcal{T}_{c}$ is a lifetime factor giving the probability that a cluster of mass $m_{c}^{2}$ decays within a time interval $\Delta t$ in the global frame, here the ep c.m.s.,

$$
\mathcal{T}_{c}\left(E_{c}, m_{c}^{2}\right)=1-\exp \left(-\frac{\Delta t}{t_{c}\left(E_{c}, m_{c}^{2}\right)}\right),
$$

with the Lorentz-boosted lifetime $t_{c}=\gamma_{c} \tau_{c} \simeq E_{c} / m_{c}^{2}$. In this scheme, a particular cluster decay mode is obtained from Eq. (31) by summing over all possible decay channels, weighted with the appropriate spin, flavor, and phase-space factors, and then choosing the actual decay mode according to the relative probabilities of the channels.

b. Beam clusters. The fragmentation of the beam cluster containing the spectator partons mimics in our model what is commonly termed the "soft underlying event," namely, the emergence of those final-state hadrons that are associated with the nonperturbative physics which underlies the perturbatively accessible dynamics of the hard interaction with parton-shower fragmentation.

In the spirit of Ref. [34], we employ a (suitably modified for our purposes) version of the soft hadron production model of the UA5 Collaboration [40], which is based on a parametrization of the CERN $p \bar{p}$ collider data for minimumbias hadronic collisions. The parameters involved in this model are set to give a good agreement with those data.

We view soft hadron production as a universal mechanism [41] that is common to all high-energy collisions that involve beam hadrons in the initial state, and that depends essentially on the total energy momentum of the fragmenting final-state beam remnant. Accordingly, we assume that the fragmentation of the final-state beam cluster depends solely on its invariant mass $M$, and that it produces a charged, particle multiplicity with a binomial distribution [40],

$$
P(n)=\frac{\Gamma(n+k)}{n ! \Gamma(k)} \frac{(\bar{n} / k)^{n}}{(1+\bar{n} / k)^{n+k}},
$$

where the mean charged multiplicity $\bar{n} \equiv \bar{n}\left(M^{2}\right)$ and the parameter $k \equiv k\left(M^{2}\right)$ depends on the invariant cluster mass ${ }^{9}$ according to the particle data parametrization [40],

$$
\begin{aligned}
& \bar{n}\left(M^{2}\right)=10.68\left(M^{2}\right)^{0.115}-9.5, \\
& k\left(M^{2}\right)=0.029 \ln \left(M^{2}\right)-0.064 .
\end{aligned}
$$

\footnotetext{
${ }^{9}$ Notice that in our model $M$ fluctuates statistically, as a result of fluctuations of the initial-state parton configuration in the proton and the variation of the hard scattering variables $x$ and $Q^{2}$. Hence, the distribution (33) and the mean multiplicity (34) vary from event to event. This is in contrast with the original UA5 model, in which the fixed beam energy $\sqrt{s} / 2$ controls the energy dependence of soft hadron production.
}

Adopting the scheme of Marchesini and Webber [34], the fragmentation of a beam cluster of mass $M$ proceeds then as follows: First, a particle multiplicity $n$ is chosen from Eq. (33), and the actual charged, particle multiplicity is taken to be $n$ plus the modulus of the beam cluster charge. Next, the beam cluster is split into subclusters $\left(q_{1} \overline{q_{2}}\right),\left(q_{2} \overline{q_{3}}\right), \ldots$, $\left(q_{i}=u, d\right)$, which are subsequently hadronized in the beamcluster rest frame, in the same way as the parton clusters described in the preceding subsection. To determine the subcluster momenta, we assume a mass distribution

$$
P(M)=c(M-1) \exp [-a(M-1)]
$$

with $c$ a normalization constant and $a=2 \mathrm{GeV}^{-1}$, resulting in average value of $\langle M\rangle \approx 1.5 \mathrm{GeV}$. The transverse momenta are taken from the distibution

$$
P\left(p_{\perp}\right)=c^{\prime} p_{\perp} \exp \left[-b \sqrt{p_{\perp}^{2}+M^{2}}\right],
$$

with normalization $c^{\prime}$ and slope parameter $b=3 \mathrm{GeV}^{-1}$, and the rapidities $y$ are drawn from a simple flat distribution $P(y) \propto$ const with an extent of 0.6 units and Gaussian tails with 1 unit standard deviation at the ends. Finally, all hadronization products of the subclusters are boosted from the rest frame of the original beam cluster back into the global frame, i.e., the $e p$ c.m.s.

\section{MODEL RESULTS FOR NONDIFFRACTIVE DIS AT HERA}

\section{A. Characteristic evolution of small- $x$ vs large- $x$ scattering events}

The kinematics of DIS has very different consequences in the small- $x$ and large- $x$ regime, as we shall discuss now within our model. Specifically, we distinguish here and in the following the two distinct regimes

$$
\begin{gathered}
\text { "small" } x: 1.7 \times 10^{-4} \leqslant x \leqslant 2.3 \times 10^{-3} \\
\text { "large" } x: x>5 \times 10^{-3},
\end{gathered}
$$

where the small- $x$ regime is the typical range probed at HERA, and part of the large- $x$ range $\left(x \geqslant 5 \times 10^{-2}\right)$ corresponds to previous fixed-target experiments (cf. Fig. 3). Table I provides the corresponding mass $W$ of the hadronic system (equal to the total hadronic energy in the $\gamma p$ c.m.s.), where the small- $x$ regime is the HERA range which we primarily focus on in the following.

Figure 6 illustrates vividly the differences between the small- $x$ (left panel) and large- $x$ (right panel) kinematics for typical HERA values of $Q^{2}=8 / 14 / 28 \mathrm{GeV}^{2}$. The top plots show the associated probability distributions for the occurrence of a particular mass $W$ of the hadronic system produced by the hard interaction in the specified $Q^{2}$ and $x$ range. As could be expected, the differences between the two $x$ domains are striking: not only the shape, but also the mean values of the distributions are very distinct (note the different scales of the $W$ axis). The most probable $W$ values lie between 100-200 GeV (small $x$ ) and between 10-20 GeV (large $x$ ). The plots in the middle show the $W$ dependence of the total hadron multiplicity calculated within our model. The shape of the curves is again rather different, which is a 


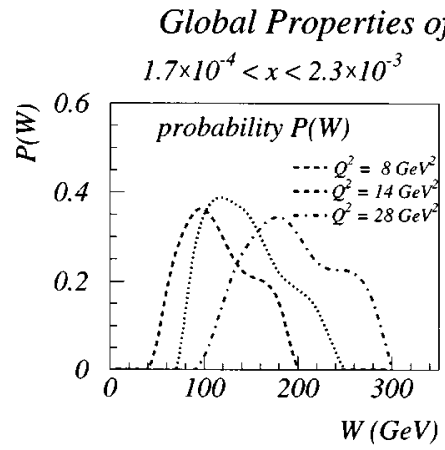

small / large $x$ regions
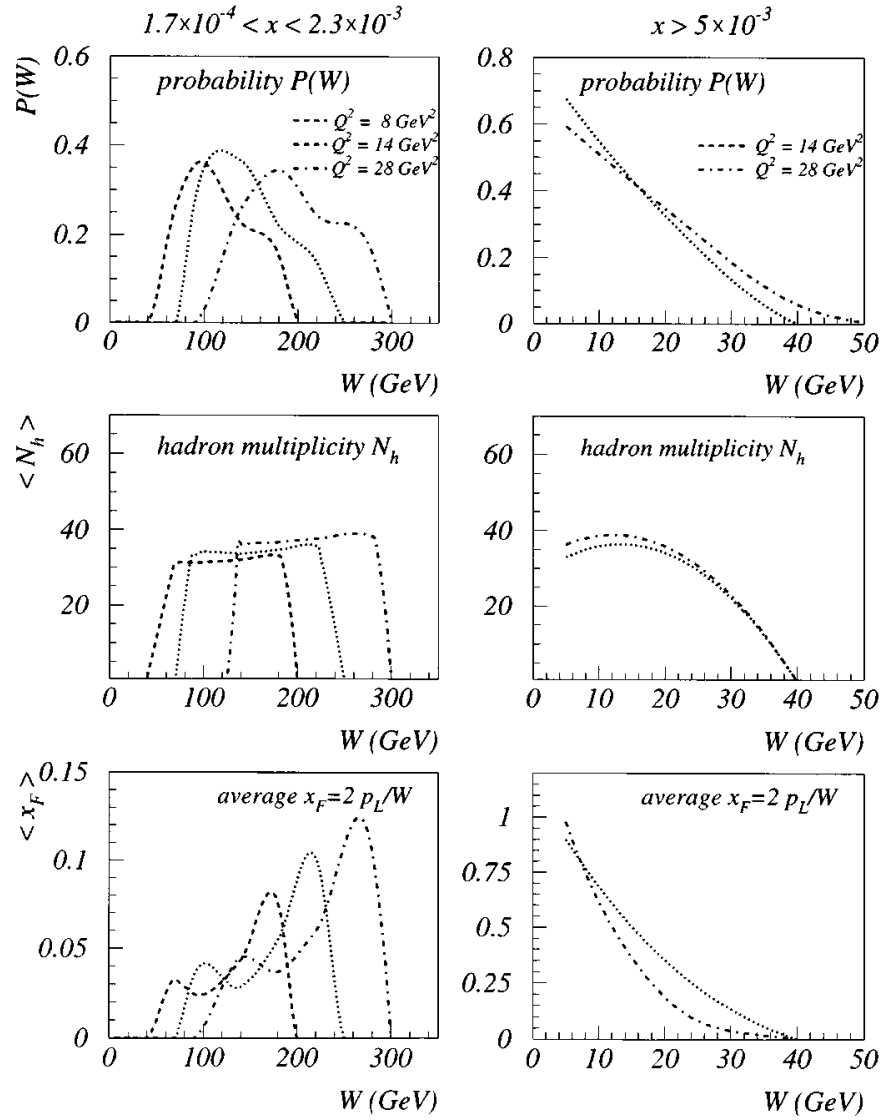

FIG. 6. Characteristic differences in global properties between "small"' $x$ (left panel) and "large"'- $x$ DIS events (right panel), as defined in Eq. (37). The two ranges refer to the values of Bjorken $x$ of the quark struck by the photon with selected $Q^{2}$. The small- $x$ range is typical for the kinematics of HERA experiments, whereas the large- $x$ regime corresponds to the phase-space region probed by previous fixed-target experiments. Compared are (a) the probability distributions for the production of a hadronic system of mass $W$ (top), (b) the corresponding $W$ dependence of the total hadron multiplicity $N_{h}$ (middle), and (c) the resulting mean values of $x_{F}=2 p_{z} / W$, with $p_{z}$ in the ep c.m.s. along the beam axis in the opposite direction to the incoming proton.

direct consequence of the probability distribution in $W$, and the phase space available for a given $W$. On the other hand, the total numbers of produced hadrons $\left(N_{h} \simeq 35-40\right)$ in events around the most probable $W$ are very similar. The bottom plots show the corresponding mean values of the $x_{F}=2 p_{z} / W$, with $p_{z}$ in the ep c.m.s. along the beam axis in the opposite direction to the incoming proton, i.e., the fractional longitudinal momentum carried by the final-state hadrons which emerge from the parton shower and fragmentation of the struck quark jet. Again, the $W$ dependences of $\left\langle x_{F}\right\rangle$ are very different in the small- $x$ and large- $x$ regimes, with the typical $x_{F}$ ranges $0.03 \leqq\left\langle x_{F}\right\rangle \lesssim 0.13$ and $\left\langle x_{F}\right\rangle \gtrsim 0.5$, respectively.

The distinct characters of small- $x$ and large- $x$ DIS events in the kinematic regimes discussed above are accompanied by different space-time evolution patterns of the particles in position and momentum space. As explained in Sec. III, our approach allows us to follow the time rate of change of the
Time evolution of particle spectra
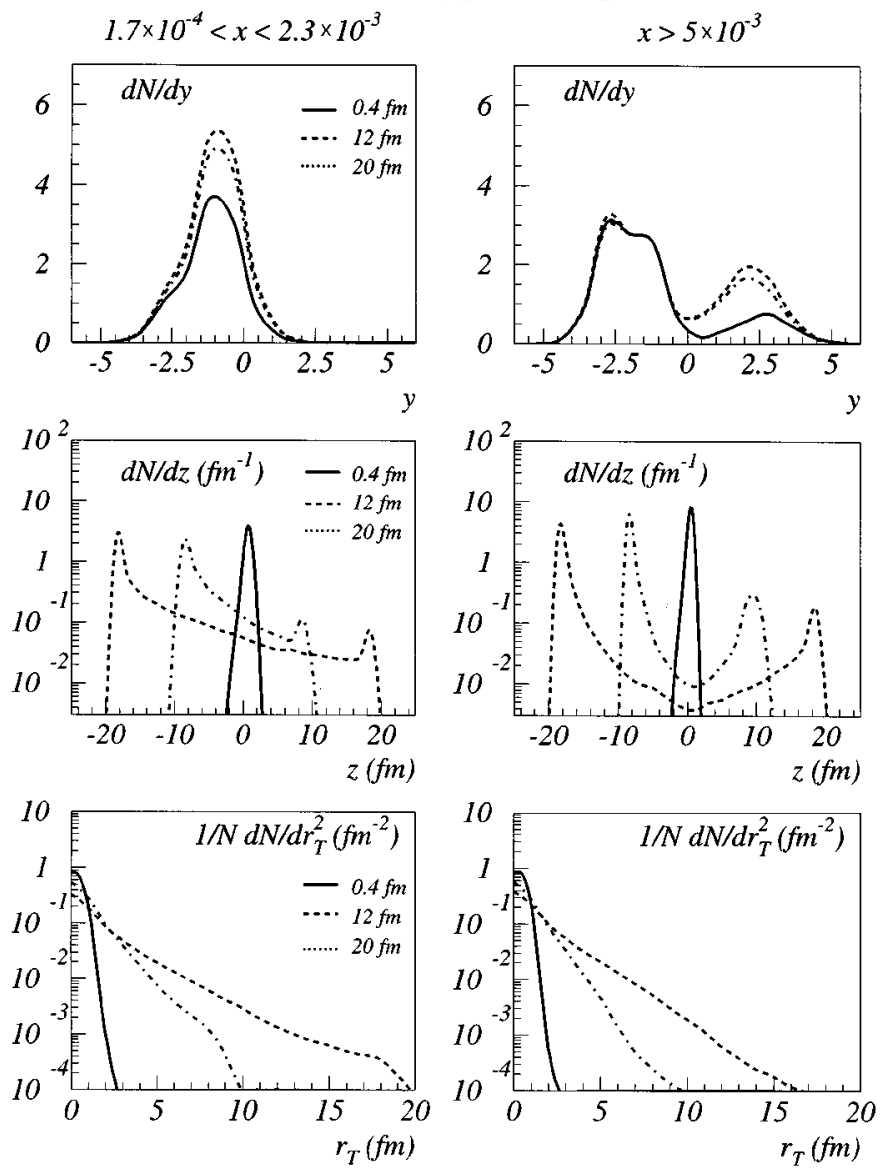

FIG. 7. Characteristic differences in the space-time evolution pattern of "small" $-x$ (left panel) and "large"' $x$ DIS events for DIS events at $Q^{2}=28 \mathrm{GeV}$ (in correspondence with Fig. 6). Compared at three different times $t=0.4 / 12 / 20 \mathrm{fm} / \mathrm{c}$ are (a) the rapidity distribution $d N / d y$ (top), (b) the particle distribution along the ep beam axis, $d N / d z$, and (c) the particle distribution perpendicular to the beam axis, $1 / N d N / d r_{\perp}$. Note that the distributions include all particle species (partons and hadrons) present in the system at the quoted given times. However, the $y$ distributions only count the secondary particles and exclude the primary partons of the original proton, beacuse their rapidity is not well defined, whereas the $z$ and $r_{\perp}$ spectra include also those primary partons.

particle densities and associated spectra. Figure 7 exhibits the time evolution of the rapidity $(y)$ distribution, and the particle distributions in longitudinal $(z)$ and transverse $\left(r_{\perp}\right)$ direction with respect to the ep c.m.s., our chosen global frame of description. The left (right) panel corresponds to small- $x$ (large- $x$ ) events with fixed $Q^{2}=28 \mathrm{GeV}^{2}$. In each plot the three curves correspond to times $0.4 / 12 / 20 \mathrm{fm} / c$ after the photon-quark scattering, and each curve includes all particles (partons and formed hadrons), which are actively present in the mixed particle system at the specified times. Comparing the time development of the spectra $d N / d y$ spectra for small- $x$ and large- $x$ events, one observes that in the former event-type most particles are produced at central rapidities $|y| \leqslant 1$, with a shift toward the proton side (negative $y$ ), while in the latter event class this region is least populated. Related to that, the $d N / d z$ distributions show a much 
larger particle production along the beam axis around $z=0$ $\mathrm{fm}$ for small- $x$ than that for large- $x$ events. From the $1 / N d N / d r_{\perp}^{2}$ distribution one can see that for small- $x$ scattering the diffusion of the expanding particle system in the transverse direction is faster than that in the large- $x$ events, an effect that arises from the transverse pressure of the larger number of produced particles in the central region.

\section{B. Inclusive hadron spectra in $x_{F}, p_{T}^{*}$ and the $\left\langle p_{T}^{* 2}\right\rangle$ dependence}

The study of particle multiplicities and momentum distributions of the hadronic final state at HERA provides sensitive information about both the QCD processes at the parton level and the properties of hadron formation. An excellent recent review can be found in Ref. [42]. One of the attractive features of the HERA experiments is the production of a large-mass hadronic final state with $W \simeq 100-250 \mathrm{GeV}$ (an order of magnitude larger than those in previous fixed-target experiments). The conjecture is, therefore, that the influence of the nonperturbative QCD effects is less important and that, in the spirit of "local parton-hadron duality" [43], the observed hadronic final state reflects more the dynamics of the partonic processes.

Particularly sensitive measures of the parton level dynamics are the $x_{F}$ and $p_{\perp}^{*}$ distributions, as well as the $\left\langle p_{\perp}^{* 2}\right\rangle$ of produced charged hadrons, as measured in the $\gamma p$ c.m.s. (5), where the Feynman variable $x_{F}=2 p_{\|}^{*} / W$ and $p_{\perp}^{*}$ characterize the momentum components of hadrons parallel and transverse to the photon direction. At large values of $W$ (small values of Bjorken $x$ ), these observables are sensitive to hard multigluon radiation. This feature is evident in Fig. 8, where we plot our model results for the $x_{F}$ and $p_{\perp}^{*}$ spectra and the dependence of $\left\langle p_{\perp}^{* 2}\right\rangle$, for three typical HERA values $Q^{2}=8 / 14 / 28 \mathrm{GeV}^{2}$ and the small- $x$ regime defined by Eq. (37). Also shown are the corresponding measured distributions for $Q^{2}=28 \mathrm{GeV}^{2}$ from ZEUS [24], with which the calculated dashed-dotted curves $\left(Q^{2}=28 \mathrm{GeV}^{2}\right)$ agree reasonably well. All three distributions have a specific form due to QCD gluon emission on the parton level which cannot be explained by the naive "quark-parton model" (QPM) which accounts only for the lowest-order photon-quark scattering and omits all higher-order QCD radiation. For comparison, the QPM results are plotted as thin curves.

The $x_{F}$ distributions (top) show steep exponential decreases above $x_{F} \approx 0.05-0.1$ and an enhanced particle yield below that value. Note that the QPM, i.e., the leading-order Born scattering alone, gives a slightly shallower decrease. The effect of the higher-order radiative processes is, however, very prominent in the $p_{\perp}^{*}$ spectra (middle) integrated over $x_{F} \geqslant 0.05$, which show a power-law dependence due to multigluon emission and a significant contribution of hard gluons with transverse momenta $\gtrsim 3 \mathrm{GeV}$ (large, in view of $\sqrt{Q^{2}}=3-5 \mathrm{GeV}$ ). This result is in vivid contrast with the corresponding QPM result, which hardly gives any transverse momenta $\gtrsim 1 \mathrm{GeV}$. The mean square of $p_{\perp}^{*},\left\langle p_{\perp}^{* 2}\right\rangle$ (bottom) is particularly sensitive to the tail of the distribution and exhibits half of a "sea-gull" shape for positive values of $x_{F}$. The rise of $\left\langle p_{\perp}^{* 2}\right\rangle$ with increasing $x_{F}$ is due to the leading hadron effect, which can be understood as follows: If
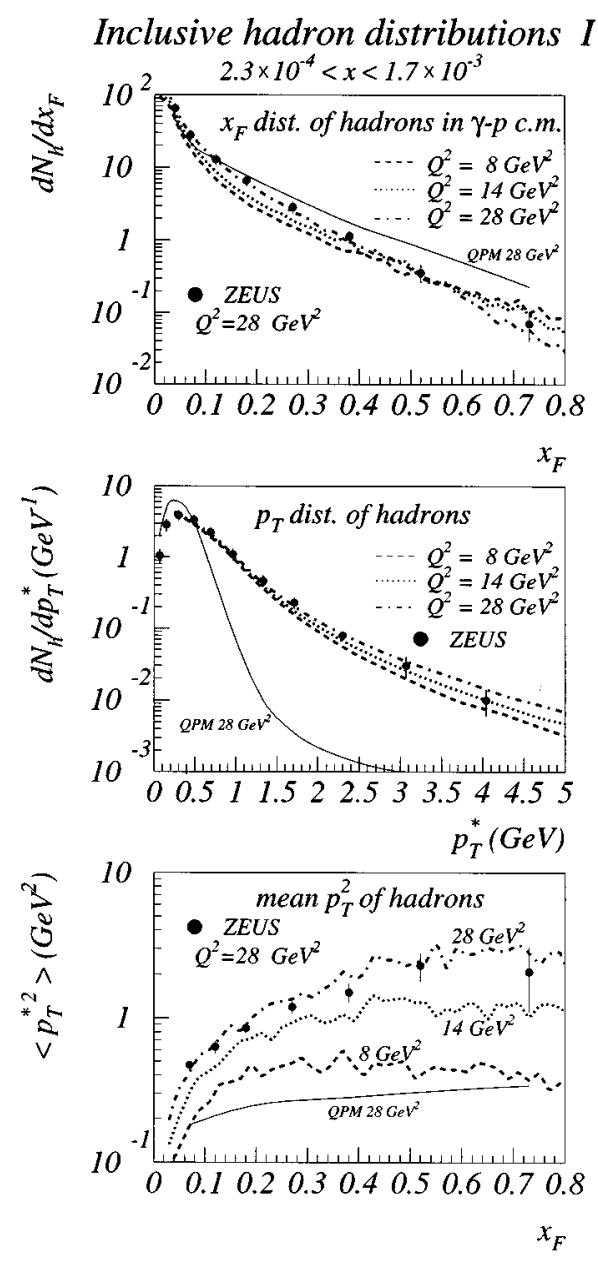

FIG. 8. Model results for differential charged hadron multiplicities with respect to the $\gamma p$ c.m.s. as a function of $x_{F}$ and $p_{\perp}^{*}$ for $x_{F}>0.05$, as well as $\left\langle p_{\perp}^{* 2}\right\rangle$ as a function of $x_{F}$. We compare results for the small- $x$ regime (37) at typical HERA values of $Q^{2}=8 / 14 / 28 \mathrm{GeV}^{2}$. The data points are measured distributions from ZEUS [43] for $Q^{2}=28 \mathrm{GeV}^{2}$, and the thin solid curves represent the corresponding expectations of the "naive" quark parton model (QPM).

$z_{h}$ denotes the fraction of momentum the initially struck quark transferred to a hadron,

$$
\left\langle p_{\perp}^{* 2}\right\rangle=z_{h}\left(\left\langle p_{\perp}^{* 2}\right\rangle_{\text {prim }}+\left\langle p_{\perp}^{* 2}\right\rangle_{\text {sec }}\right)+\left\langle p_{\perp}^{* 2}\right\rangle_{\text {frag }},
$$

where $p_{\perp \text { prim }}^{*}$ is the primordial transverse momentum of the quark to which the photon couples [cf. Eq. (7)], $p_{\perp \text { sec }}$ is the secondary contribution from QCD radiation, and $p_{\perp}$ frag denotes the additional transverse momentum produced in the fragmentation and hadron formation process, which is in the average about $0.45 \mathrm{GeV}$ and almost independent of $W$ and $Q^{2}$. From Eq. (38) one sees that the leading hadrons at large $x_{F}$, which carry higher fractional momentum $z_{h}$, also carry a higher fraction of the actual parton transverse momentum (primary plus secondary). This explains qualitatively the "sea-gull" shape and the rise of the $\left\langle p_{\perp}^{* 2}\right\rangle$ of hadrons as a function of $x_{F}$. Furthermore, one observes that the more gluons with relatively large transverse momentum are radiated, the larger the contribution to $\left\langle p_{\perp}^{* 2}\right\rangle_{\text {sec }}$, and hence the stronger is the effect. In the QPM with no QCD radiation at 


\section{Mean squared transverse momentum}
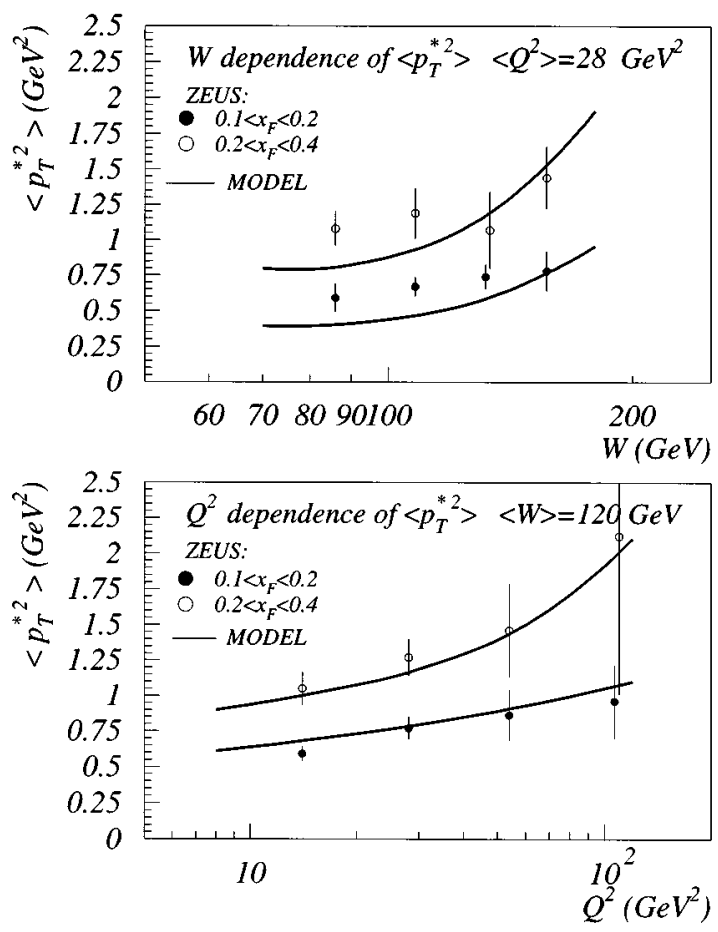

FIG. 9. Model results for the $W$ dependence for fixed $Q^{2}=28$ $\mathrm{GeV}^{2}$ and the $Q^{2}$ dependence for fixed $W=120 \mathrm{GeV}$ of the mean squared transverse momentum of charged hadrons $\left\langle p_{\perp}^{* 2}\right\rangle$. The plots refer to the $\gamma p$ c.m.s. and separate the two intervals $0.1<x_{F}<0.2$ and $0.2<x_{F}<0.4$. The data points are from the ZEUS experiment [43].

all, the total $\left\langle p_{\perp}^{* 2}\right\rangle$ is, therefore, a factor of 5-10 smaller and has only a weak $x_{F}$ dependence.

In Fig. 9 the $W$ and $Q^{2}$ dependences of the mean squared $p_{\perp}^{*}$ of hadrons is shown for two intervals $0.1<x_{F}<0.2$ and $0.2<x_{F}<0.4$, and compared with data obtained by the ZEUS Collaboration. The agreement with the data is fairly good for the $Q^{2}$ dependence, whereas it is less clear for the dependence on $W$. The $\left\langle p_{\perp}^{* 2}\right\rangle$ depends strongly on both $W$ (for fixed $Q^{2}=28 \mathrm{GeV}^{2}$ ) and $Q^{2}$ (for fixed $W=120 \mathrm{GeV}$ ). It is worth noting that in previous fixed-target experiments at lower energies the $\left\langle p_{\perp}^{* 2}\right\rangle$ is generally much smaller, depending only weakly on $W$ (however, at much smaller $W$ values), and essentially flat in $Q^{2}$.

We remark that the new class of diffractive events with a large rapidity gap is measured [24] to have very different $p_{\perp}^{*}$ spectrum and $\left\langle p_{\perp}^{* 2}\right\rangle$ from the nondiffractive events that we have just discussed. The particle distribution in $p_{\perp}^{*}$ of diffractive events falls much steeper and resembles closely the QPM curve in the middle plot of Fig. 8. The mean values $\left\langle p_{\perp}^{* 2}\right\rangle$ are smaller by a factor of $2-5$, and lie just slightly above the QPM curve in the bottom plot of Fig. 8. This indicates that diffractive events resemble in a way DIS events with very little QCD radiation, consistent with the common interpretation that the photon couples in these events to the proton via a colorless intermediate state which does not fragment by multiparton emission.
Inclusive hadron distributions II
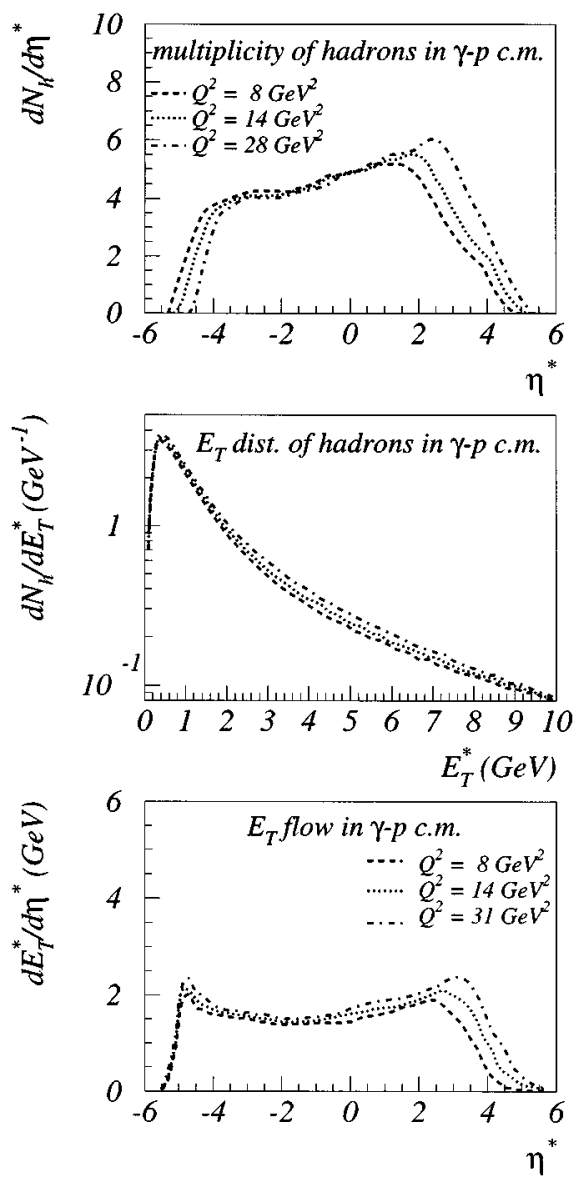

FIG. 10. Hadron distributions in the $\gamma p$ c.m.s. as a function of pseudorapidity $\eta^{*}$ and of transverse energy $E_{\perp}^{*}$, as well as the $E_{\perp}^{*}$ flow vs pseudorapidity. The model results refer to $Q^{2}=8 / 14 / 28 \mathrm{GeV}^{2}$ and $2.3 \times 10^{-4} \leqslant x \leqslant 1.7 \times 10^{-3}$ corresponding to $W>60 / 90 / 130 \mathrm{GeV}$.

\section{Transverse energy flow}

Whereas the inclusive $x_{F}$ and $p_{\perp}$ distributions of produced hadrons are sensitive to the multijet structure due to hard-gluon radiation as discussed above, the analysis of inclusive hadron distributions in terms of the global energy flow extends to classes of events which cannot be identified unambiguously as $n$-jet events. According to the idea of "local parton-hadron duality' [43], the pattern of overall distribution of energy among the partons in an event determines the energy flow observed in the hadronic spectrum. The energy flow $d E_{\perp}^{*} / d \eta^{*}$, and similarly the particle flow $d N_{h} / d \eta^{*}$, are commonly studied as a function of pseudorapidity $\eta^{*}$ in the hadronic center-of-mass system, i.e., the $\gamma p$ c.m.s., where

$$
\eta^{*}=-\ln \left(\tan \frac{\theta^{*}}{2}\right)=\ln \left(\frac{E^{*}+p_{\|}^{*}}{p_{\perp}^{*}}\right), \quad E_{\perp}^{*}=\sqrt{E^{* 2}+p_{\perp}^{* 2}},
$$

with $\theta^{*}, p^{*}, p_{\perp}^{*}$ defined with respect to the photon direction.

In Fig. 10 we show model results for the distribution of 
hadrons in $\eta^{*}, E_{\perp}^{*}$, and as well as in the $E_{\perp}^{*}$ flow. As before, we choose $Q^{2}=8 / 14 / 28 \mathrm{GeV}^{2}$ for the small- $x$ regime $2.3 \times 10^{-4} \leqslant x \leqslant 1.7 \times 10^{-3}$ corresponding to $W>60 / 90 / 130$ $\mathrm{GeV}$ (cf. Table I). The plotted distributions reflect the typical event geography that one expects already from QPM considerations. Recall that in the QPM, with the neglect of higherorder QCD corrections, the struck quark and the proton remnant system each carry an energy $W / 2$ in the $\gamma p$ c.m.s. and move back to back with rapidities $\pm y_{\max }^{*} \propto \pm \ln W / m_{\pi}$. The fragmentation of the two receding charges fills the intermediate pseudorapidity region with hadrons. ${ }^{10}$ The width of the hadron distribution in the final state is proportional to $\ln W$, while its height is approximately independent of $W$. The width of the quark jet and the proton fragmentation region bounded by $\pm \eta_{\max }^{*}$ is typically about 2 units, corresponding to the $x_{F}$ range $\left|x_{F}\right|>0.05$. Hence, at high values of $W$ (small $x$ ), the pseudorapidity range populated by hadrons can be divided in three regions: (i) the current jet region from $\left(\eta_{\max }^{*}-2\right)$ to $\eta_{\max }^{*}$ (ii) the proton fragmentation region from $\left(-\eta_{\max }^{*}+2\right)$ to $-\eta_{\max }^{*}$, and (iii) a central plateau region in between.

The pseudorapidity distribution $d N_{h} / d \eta^{*}$ (Fig. 10, top), as calculated in our model, shows a distorted version of the naive QPM picture due to the higher-order QCD radiation effects. The spectrum is asymmetric and the central plateau is rising from the proton fragmentation region to the current jet region, rather than being flat. Particularly different from the QPM is the behavior in the current jet region $\eta^{*} \geq 3$, which shows a clear increase with $Q^{2}$ of both the height and the width of this part of the hadron distribution, an effect of the jet broadening due to gluon radiation off the struck quark.

The transverse energy distribution $d N_{h} / d E_{\perp}^{*}$ (Fig. 10, middle) is naturally similar to the $p_{\perp}^{*}$ spectrum discussed before (cf. Fig. 8). It again exhibits a power-law behavior that is characteristic for gluon emission with significant $p_{\perp}^{*}$ a feature which becomes more prominent with increasing $Q^{2}$, because of the enlarged phase space and extended duration of parton-shower activity before hadronization.

The hadronic energy flow $d E_{\perp}^{*} / d \eta^{*}$ (Fig. 10, bottom) mirrors the distribution of energy and transverse momentum among the final-state particles in a similar way to the pseudorapidity distribution discussed above. The characteristic features are: first, a central plateau with a slight dip and a height almost independent of $Q^{2}$, second, an increase with $Q^{2}$ of energy deposit in the current jet region around $\eta^{*} \approx 3$, resulting from radiation of the timelike shower of the quark after the hard scattering, and third, a similar though much less significant increase with $Q^{2}$ of the activity around $\eta^{*} \approx-5$ in the proton fragmentation region due to radiation from the spacelike shower before the hard interaction, which is going along the proton direction and opposite to the timelike radiation.

In Fig. 11 the $x$ (or equivalently, $W$ ) dependence of the $E_{\perp}^{*}$ flow in the current jet hemisphere in the $\gamma p$ c.m.s. is investigated for fixed $Q^{2}=28 \mathrm{GeV}$. We plot $d E_{\perp}^{*} / d \eta^{*}$ for

\footnotetext{
${ }^{10}$ For the purposes of this discussion, we will now neglect the difference between rapidity $y$ and pseudorapidity $\eta$.
}
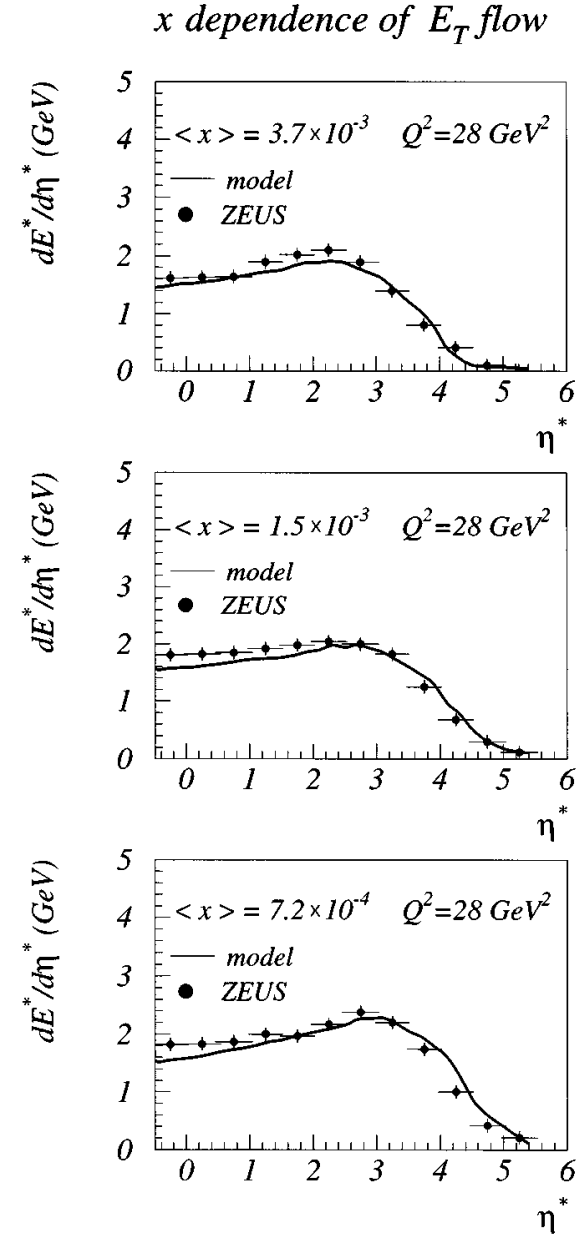

FIG. 11. The $x(W)$ dependence of the $E_{\perp}^{*}$ flow on the current jet side in the $\gamma p$ c.m.s. at $Q^{2}=28 \mathrm{GeV}$ and for $\langle x\rangle=3.7 \times 10^{-3} / 1.5 \times 10^{-3} / 7.2 \times 10^{-4}, \quad$ corresponding to $W \simeq 78 / 137 / 197 \mathrm{GeV}$. The experimental data are from ZEUS [44].

three different $x$ ranges with average values $\langle x\rangle=3.7 \times 10^{-3} / 1.5 \times 10^{-3} / 7.2 \times 10^{-4}$, corresponding to $W \simeq 78 / 137 / 197 \mathrm{GeV}$. Also depicted are the data points of the measured distributions from the ZEUS collaboration [44]. Although the model slightly underestimates the data around $\eta^{*}=0$, qualitative conclusions that may be drawn are: first, the height of the plateaulike region for $\eta^{*} \lesssim 1.5$ is rather independent of $x$, and second, with decreasing $\langle x\rangle$ (from top to bottom) the peak around the current jet moves visibly towards larger rapidities, with $\eta_{\text {peak }}^{*} \simeq 2.4 / 2.8 / 3.2$, respectively, while the height of the peak appears to be stable.

\section{Mass distributions of the observed hadronic final state}

As mentioned in the introduction, a new class of DIS events is observed at HERA in the experiments by ZEUS [5] and $\mathrm{H} 1$ [6], events which are characterized by a largerapidity gap (LRG) between the proton and the rest of the hadronic final state that is measured in the detector. The properties of these events indicate a diffractive production mechanism via exchange of a coherent colorless object between the photon and the proton [cf. Fig. 1(b)], accompanied by a suppression of QCD radiative processes, which are, as we discussed, so prominent in nondiffractive events [cf. Fig. 
1(a)] with no rapidity gap (NRG). Because in the experiment both diffractive LRG and nondiffractive NRG events are mixed (with a relative contribution of $\approx 5-10 \%$ from LRG events), the determination of the diffractive cross section requires the detailed knowledge and subtraction of the nondiffractive contribution.

A method to separate diffractive and nondiffractive contributions suggested by ZEUS [45] uses the mass $M_{X}$ of the hadronic system $X$ that is measured in the detector, where

$$
\begin{aligned}
M_{X}^{2} \equiv & \left\{\left(\sum_{j} E_{j}\right)^{2}-\left(\sum_{j} p_{x_{j}}\right)\right. \\
& \left.-\left(\sum_{j} p_{y_{j}}\right)^{2}-\left(\sum_{j} p_{z_{j}}\right)^{2}\right\}_{\text {detector }} \\
< & W^{2}
\end{aligned}
$$

includes all observed particles except the outgoing electron. Because of the finite resolution and geometric acceptance of the detector, $M_{X}$ is naturally smaller than the total invariant mass $W$, with the event fluctuations giving rise to a distribution in $M_{X}$. The remarkable feature of the distributions in $M_{X}^{2}$ and $\ln M_{X}^{2}$ is that they exhibit very different behavior for the two event-types and are sensitive measures of the event structures. In this context, we investigate in the following the $M_{X}$ spectrum of purely nondiffractive NRG events and its $Q^{2}$ and $x$ (or $W$ ) dependences, so as to provide an estimate of the nondiffractive contribution underlying the diffractive LRG component.

Let us briefly summarize the state of knowledge in order to set the stage. As illustrated in Fig. 1(b), in diffractive scattering the outgoing proton or low-mass nucleonic system remains colorless and escapes through the forward beam hole, while the system $X$ from the dissociation of the photon is, in general, almost fully contained in the detector. Diffractive dissociation prefers small $M_{X}$ values $\left(\ln M_{X}^{2} \lesssim 4\right)$ with an event distribution of the form [45]

$$
\frac{d \mathcal{N}^{\text {diff }}}{d \ln M_{X}^{2}}=a\left(\frac{1}{M_{X}^{2}}\right)^{n},
$$

where at high energy one expects $n \approx 0$ [41], approximately independent of the total $\gamma p$ c.m. energy $W$.

On the other hand, in nondiffractive events the incident proton is broken up and the remnant of the proton is a colored object with the struck quark taking away the net color. As we discussed before, this results in a substantial amount of initial- and final-state radiation followed by hadron formation between the directions of the proton and the current jet [cf., Fig. 1(a)]. From perturbative QCD arguments, as well as simple phase-space considerations, one expects [45] that the associated event distributions are peaked at large $M_{X}$ values $\left(\ln M_{X}^{2} \approx 5-10\right)$ with an exponential falloff towards smaller

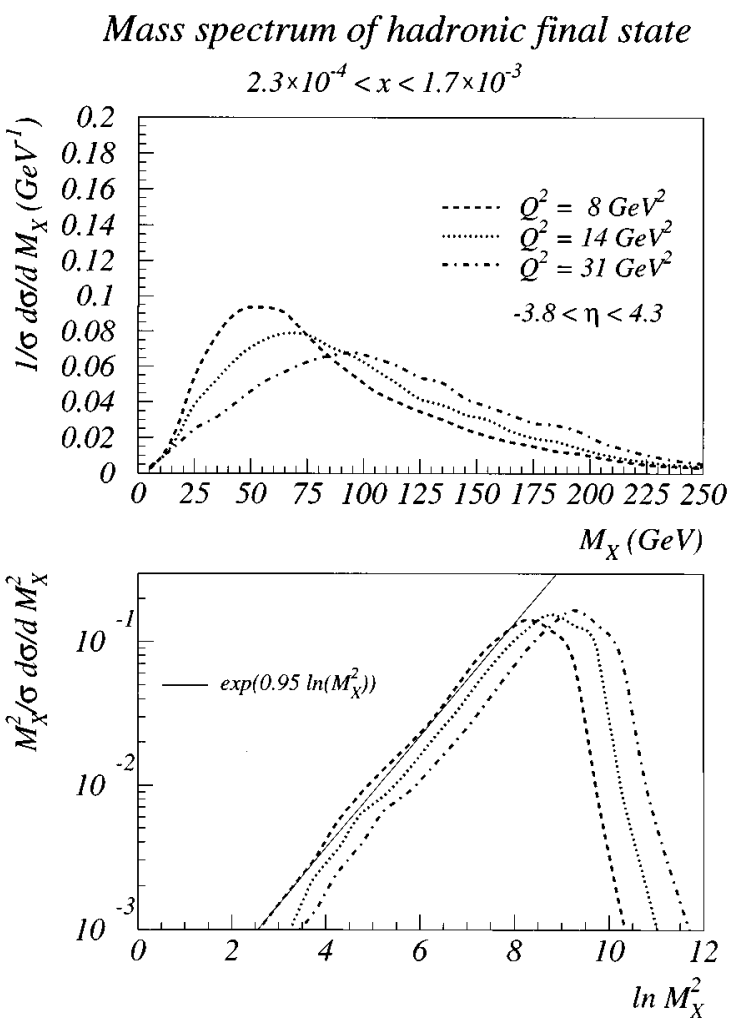

FIG. 12. Comparison of normalized event distributions in $M_{X}$ (top) and $\ln M_{X}^{2}$ (bottom) at $Q^{2}=8 / 14 / 28 \mathrm{GeV}^{2}$, and for $2.3 \times 10^{-4} \leqslant x \leqslant 1.7 \times 10^{-3}$, with $-3.8 \leqslant \eta \leqslant 4.3$.

masses, ${ }^{11}$

$$
\frac{d \mathcal{N}^{\text {nondiff }}}{d \ln M_{X}^{2}}=c \exp \left(b \ln M_{X}^{2}\right),
$$

where $c$ is a constant. The slope $b$ is the parameter of interest: on the parton level it can be shown to be determined by the QCD Sudakov form factor and thus the probability for gluon emission. In our model for parton-hadron conversion, it is, therefore, closely related to the probability for cluster formation and hadron production.

In Fig. 12 we show the nondiffractive event distributions in $M_{X}$ (top) and $\ln M_{X}^{2}$ (bottom). The plots compare calculations with the fixed values $Q^{2}=8 / 14 / 28 \mathrm{GeV}^{2}$, normalized to the total number of events, and within the nominal kinematic acceptance of the ZEUS detector, $-3.8 \leqslant \eta \leqslant 4.3$. The distribution $1 / \mathcal{N} d \mathcal{N} / d M_{X}\left(\mathcal{N} \equiv \mathcal{N}^{\text {nondiff }}\right)$ in the top part of the figure exhibits a clear $Q^{2}$ dependence in both the position of the peak and the extension of the tail towards large $M_{X}$ values. The mean values $\left\langle M_{X}\right\rangle=78 / 93 / 105 \mathrm{GeV}$ for $Q^{2}=8 / 14 / 28 \mathrm{GeV}^{2}$. The properties of the distributions are most evident when studied as a function of $\ln M_{X}^{2}$ as in the

\footnotetext{
${ }^{11}$ Another salient feature of the $\ln M_{X}^{2}$ distribution is an observed scaling in $\ln M_{X}^{2}-\ln W^{2}$ [45], implying that the position of the highmass peak in $\ln M_{X}^{2}$ grows proportional to $\ln W^{2}$, and the slope of the exponential fall off to small $\ln M_{X}^{2}$ values is approximately independent of $W$.
} 
$W$ dependence of $\ln \left(M_{X}^{2}\right)$ spectra
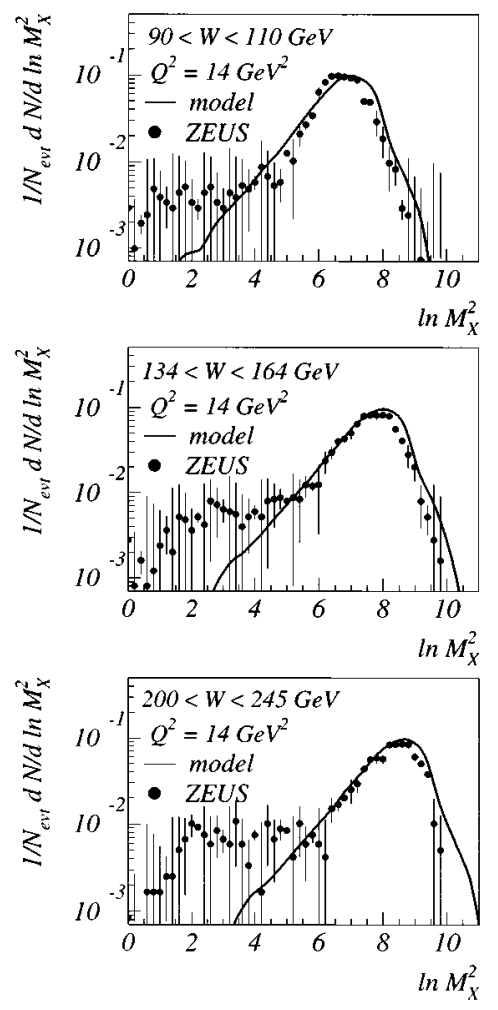

FIG. 13. Model results for the $W$ dependence of the $\ln M_{X}^{2}$ distribution at $Q^{2}=14 \mathrm{GeV}$ for three $W$ intervals, compared with the corresponding measured distributions from ZEUS [45].

bottom part where $1 / \mathcal{N} d \mathcal{N} / d \ln M_{X}^{2}$ is shown for the same parameters. In this representation the mass peak exhibits a steep exponential fall off towards smaller $M_{X}^{2}$ values. The associated slope exhibits no significant dependence on $Q^{2}$ and comes out as $b=0.95 \pm 0.1$, when compared with the form (42) as indicated by the straight line in the plot. On the other hand, an experimental data analysis by ZEUS [45] yields a steeper slope, namely, $b^{\text {expt }}=1.46 \pm 0.15$. The discrepancy between $b^{\text {expt }}$ and $b$ can have various reasons associated with experimental effects that we did not attempt to simulate or correct for, e.g., detector acceptance or other effects, such as energy loss of particles in the calorimeter. Such effects may affect the value of $b$. However, the fact that both $b$ and $b^{\text {expt }}$ come out to be universally constant supports the conjecture that the difference between the values of $b$ and $b^{\text {expt }}$ is due to global effects that are missing in our calculations.

In Fig. 13 we investigate the $W$ dependence of the $\ln M_{X}^{2}$ distribution at $Q^{2}=14 \mathrm{GeV}$, plotting our results for three distinct intervals of the total $\gamma p$ c.m. energy $W$. One observes that the slope is the same in all three $W$ ranges, and hence, appears to be independent of $W$ as well as $Q^{2}$. The position of the peak, however, is shifted to larger values as $W$ is increased, as in the previous figure when $Q^{2}$ is increased. Also shown in Fig. 13 are the corresponding measured distributions measured by ZEUS [45], with which our model calculations agree reasonably well for the large $M_{X}$ range, $\ln M_{X}^{2} \gtrsim 4-6$, but which show an additional component at small values $\ln M_{X}^{2} \lesssim 4$. This latter is due to the diffractive (LRG) contribution, which evidently has a plateaulike (rather than exponential) shape, in agreement with the expectation (41). The comparison between the data points and our model results exhibits two important conclusions. First, the diffractive component cannot at all be explained by the standard QCD parton-shower evolution plus hadronzation model of nondiffractive events: in fact, it is completely absent therein. Second, the diffractive and nondiffractive contributions appear to be sharply separated when studied with respect to the variable $\ln M_{X}^{2}$ and allow one to subtract cleanly from the measured data sample the nondiffractive part, as calculated using this or other QCD parton-shower models.

Finally, we would like to comment on the difference between the value of $b$ calculated within our model, as compared to other parton-shower models [18,20,21], which use the string fragmentation approach [46] to hadron formation from the final-state parton ensemble. As investigated in Ref. [45], the latter give a value of $b \simeq 2$, i.e., about twice as large as in our model. We believe that this difference arises from differences in modeling the parton-hadron transition, i.e., string fragmentation vs cluster formation and decay, ${ }^{12}$ as we now discuss in more detail.

Let us first recall some general features of hadron distributions and correlations within the Mueller approach [47], where they are related by unitarity to appropriate absorptive parts of forward multiparticle scattering amplitudes. In the beam fragmentation region, which is relevant to this discussion, asymptotic properties of the single- and multiparticle distributions are controlled by Regge singularities. In particular, the asymptotic value of the single-particle density is controlled by the pomeron, with subasymptotic corrections and finite-range multiparticle correlations controlled by subleading Regge singularities. These give, in particular, local two-particle correlations with a correlation length $\Delta y=1 / \Delta \alpha$, where $\Delta \alpha$ is the difference between the intercepts at $t=0$ of the pomeron and the next subleading trajectories, commonly believed to be the $\rho$ and degenerate trajectories with $\Delta \alpha \simeq 1 / 2$, yielding $\Delta y \simeq 2$. The $\pi$ trajectory with $\Delta \alpha \simeq 1$ would yield shorter-range correlations with $\Delta y \simeq 1$. These subleading trajectories would also yield subasymptotic corrections to the $M_{X}$ distribution: $b \simeq 2 \Delta \alpha$, corresponding to $b \simeq 1(2)$ for the $\rho(\pi)$ trajectories, with the tail corresponding to rapidity-gap events corresponding to pomeron exchange with $b \simeq 0$.

With these points in mind, we now recall aspects of particle production according to the Lund string fragmentation model [46], as compared to our approach. In the former model, the string is chopped at an ordered sequence of points along the rapidity axis, with separations chosen randomly but with a mean value $\delta y \simeq 1$. The string bits then hadronize independently, with resonance decays resulting in a correlation length $\Delta y \simeq \delta y \simeq 1$. The adjacency in rapidity of the Lund fragmentation model clearly results in the minimum possible correlation length $\Delta y$, and hence, effectively to the

\footnotetext{
${ }^{12}$ The preceding parton-shower stage is essentially the same in our and other models [17], with our additional space-time information becoming relevant during cluster formation and hadronization.
} 


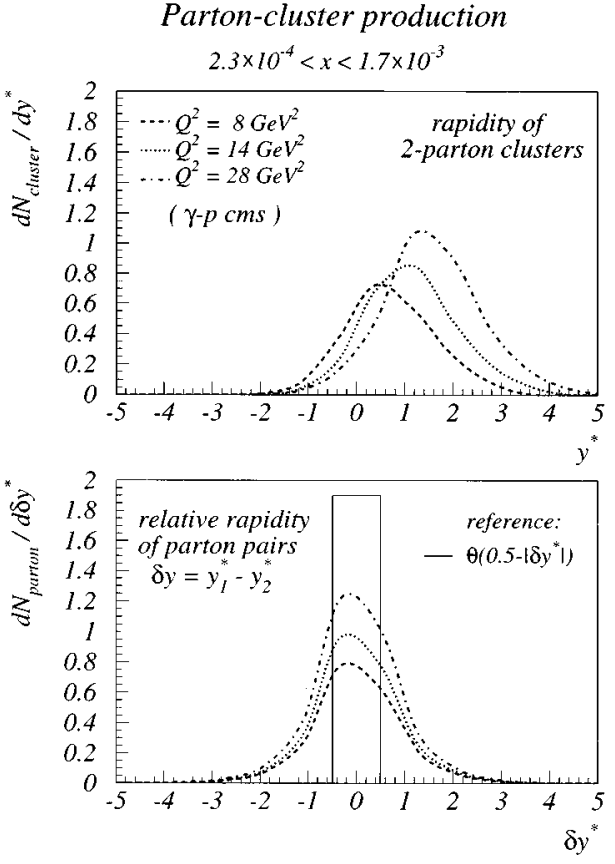

FIG. 14. Top: Population of rapidity $y^{*}$ in the $\gamma p$ c.m.s. of prehadronic clusters formed from coalesced parton pairs in the current jet region. Bottom: Distribution in relative rapidity $\delta y^{*}=y_{1}^{*}-y_{2}^{*}$ of parton pairs making up the clusters. The thin full line represents a constant rapidity separation $\delta y^{*}=1$ and serves as reference to the discussion in the text.

largest possible $\Delta \alpha$. Thus, it is no surprise to find that simulations based on this model yield a relatively high value of $b \simeq 2$, corresponding to the $\pi$ trajectory in the Mueller language. On the other hand, in our space-time approach, the prehadronic clusters are formed by adjacent pairs of partons in position space, which are not necessarily the closest in rapidity space. This point is reflected in Fig. 14, where we see that the separation in rapidity between partons that combine to form a cluster is typically $\delta y \simeq 2$, approximately a factor 2 larger than that in the Lund model. We, therefore, expect in our model that $\Delta y \simeq 2$, corresponding to $\Delta \alpha \simeq 1 / 2$ and $b \simeq 1$ as we found above.

To the extent that the experimental value of $b$ exceeds unity, it may be that our space-time approach deviates too far from the adjacency in rapidity of the Lund string fragmentation model, and the truth may well lie somewhere in between, corresponding in the Mueller approach perhaps to a combination of the $\rho$ and $\pi$ trajectories. One way to test this would be to measure experimentally the rapidity correlation length, and compare it directly with the predictions of various models. An interesting issue to watch will be whether $b$ and the effective two-particle correlation length depend on $Q^{2}$ or $W^{2}$. The naive Mueller Regge described above has been derived for incident hadrons, and may require modification at large $Q^{2}$.

\section{SUMMARY}

We have presented in this paper the application to DIS at HERA of a model for the quantum kinetics of multiparticle production that includes the space-time development of the parton shower, cluster formation, and hadronization. Compared with our previous work, novel features include tracking back to the initial proton the development of the spacelike parton-shower prior to its interaction with the virtual photon radiated by the electron. Our procedure tracks in space and time the emission and evolution during this development of timelike secondary partons, as well as the spectator partons in the proton beam fragment. ${ }^{13}$ As in our previous work, the coalescence of partons to form prehadronic clusters is determined statistically by a spatial criterion motivated by confinement and a simple nonperturbative model for hadronization.

Our space-time approach has enabled us to map the history of the particle densities and associated spectra, including the rapidity, longitudinal, and transverse distributions of particles. These hard results may be compared with intuitive pictures of the space-time development of hadronic final states in DIS. They will also form the basis for the subsequent extension of our approach to shadowing and other interesting effects in $e A$ scattering.

We have also explored in our model inclusive hadron spectra in $x_{F}$ and $p_{T}$, and the transverse energy flow. Although our model reproduces the general features of the observed pattern in energy flow, it shares with other simulations the tendency to undershoot the data around $\eta^{*}=0$. However, the discrepancy is not dramatic, and does not make a strong case for the presence of important physical effects not present in the MLLA approach we use here.

Our model provides distributions of the mass $M_{X}$ of the observed hadronic final state in events without a large rapidity gap, which can be used to estimate the background to the cross section for LRG events. We find a spectrum $\sim \exp \left(b \ln M_{X}^{2}\right)$ with an exponent $b \simeq 1$, which is not very different from the observed value $b^{\text {expt }} \simeq 1.5$. A detailed comparison with the data requires more understanding of detector effects and final-state hadron interactions, which goes beyond the scope of this paper. The value of $b$ is sensitive to the rapidity density and other properties of prehadronic clusters, so the relative success of our model, which has no parameters adjusted from its previous applications to $e^{+} e^{-}$annihilation, gives us some hope that we are capturing important aspects of this physics.

As already mentioned, the new features of our approach introduced in this paper, including the space-time treatment of the initial hadronic state, open the way to future applications of our model to $e A, p p, p A$, and $A A$ collisions, where the novel features associated with high parton densities will become more marked. We aim eventually at a unified spacetime description of all these hadronic processes.

\section{ACKNOWLEDGMENTS}

Many thanks to Mark Strikman for his helpful suggestions and critical remarks on a preliminary version of the manuscript. This work was supported in part by the U.S. DOE under Contract No. DE-AC02-76H00016.

\footnotetext{
${ }^{13}$ This machinery will be applied in the future to more complicated situations including $e A, p p, p A$, and $A A$ collisions.
} 
[1] T. Hebbeker, Phys. Rep. 217, 69 (1992).

[2] UA5 Collaboration, G. J. Alner et al., Phys. Rep. 154, 247 (1987).

[3] CTEQ Collaboration, R. Brock et al., Rev. Mod. Phys. 67, 157 (1995).

[4] W. J. Stirling, P. N. Harriman, A. D. Martin, and R. G. Roberts, in Proceedings of the Topical Workshop on the Small-x Behavior of Deep Inelastic Scattering Structure, Hamburg, Germany, 1990, edited by A. Ali and J. Bartels [Nucl. Phys. B (Proc. Suppl.) 18C, 17 (1990)]; M. Glück, E. Reya, and A. Vogt, ibid., p. 49.

[5] ZEUS Collaboration, M. Derrick et al., Phys. Lett. B 315, 481 (1993); 332, 228 (1994); 338, 438 (1994).

[6] H1 Collaboration, T. Ahmed et al., Nucl. Phys. B429, 377 (1994).

[7] M. Bengtsson and T. Sjöstrand, Z. Phys. C 37, 465 (1988); L. Durand and H. Pi, Phys. Rev. D 40, 1436 (1989); J. M. Butterworth, J. R. Forshaw, and M. Seymour, Report No. CERNTH/95-82, 1996 (unpublished).

[8] K. Geiger, Phys. Rev. D 46, 4986 (1992); 47, 133 (1993).

[9] M. Gyulassy and X.-N. Wang, Nucl. Phys. B420, 583 (1994); R. Baier, Yu. L. Dokshitzer, S. Peigné, and D. Schiff, Phys. Lett. B 345, 277 (1995); E. Levin, Report No. CBPF-NF-06195, 1995, hep-ph/9508414 (unpublished).

[10] M. Gyulassy and M. Plümer, Phys. Lett. B 234, 432 (1990); X.-N. Wang and M. Gyulassy, Phys. Rev. Lett. 68, 1480 (1992).

[11] J. Harris and B. Müller, Annu. Rev. Nucl. Part. Sci. (to be published); K. Geiger, in Quark Gluon Plasma 2, edited by R. C. Hwa (World Scientific, Singapore 1995), p. 264.

[12] E. Levin, in Deep Inelastic Scattering and Related Subjects, Proceedings of the International Workshop, Eilat, Israel, 1994, edited by A. Levy (World Scientific, Singapore, 1994).

[13] For recent experimental and phenomenological progress, see, e.g., G. Wolf, in Deep Inelastic Scattering and Related Subjects [12].

[14] J. Ellis and K. Geiger, Phys. Rev. D 52, 1500 (1995); Nucl. Phys. A590, 609 (1995).

[15] Physics at LEP 2, Proceedings of the Workshop (CERN Yellow Report No. 06-01, Geneva, Switzerland, 1996) (unpublished).

[16] J. Ellis and K. Geiger, Phys. Rev. D 54, 1967 (1996).

[17] I. G. Knowles et al., in Physics at LEP 2 [15].

[18] L. Lönnblad, Comput. Phys. Commun. 71, 15 (1992).

[19] G. Marchesini, B. R. Webber, G. Abbiendi, I. G. Knowles, M. H. Seymour, and L. Stanco, Comput. Phys. Commun. 67, 465 (1992).

[20] T. Sjöstrand, Comput. Phys. Commun. 82, 74 (1994).

[21] G. Ingelman, A. Edin, and J. Rathsman, Report No. DESY-96057, 1996, hep-ph/9605286 (unpublished).
[22] M. Bengtsson, G. Ingelman, and T. Sjöstrand, Nucl. Phys. B301, 554 (1988); A. Edin, G. Ingelman, and J. Rathsman, Report No. DESY-96-060, 1996, hep-ph/9605281 (unpublished).

[23] K. Geiger, Phys. Rev. D 54, 949 (1996).

[24] ZEUS Collaboration, M. Derrick et al., Z. Phys. C 70, 1 (1996).

[25] V. M. Belyaev and B. L. Ioffe, Nucl. Phys. B313, 647 (1989); D. Antreasyan et al., Phys. Rev. Lett. 48, 302 (1982).

[26] K. Geiger, Phys. Rep. 258, 237 (1995).

[27] Yu. L. Dokshitzer, D. I. Dyakonov, and S. I. Troyan, Phys. Rep. 58, 269 (1980); Yu. L. Dokshitzer, V. A. Khoze, A. H. Mueller, and S. I. Troyan, Rev. Mod. Phys. 60, 373 (1988).

[28] Basics of Perturbative QCD, Advanced Series on Directions in High Energy Physics, Vol. 5 (Editions Frontieres, Gif-surYvette, France, 1991).

[29] M. Glück, E. Reya, and A. Vogt, Z. Phys. C 48, 471 (1990); 67, 433 (1995)

[30] K. Konishi, A. Ukawa, and G. Veneziano, Nucl. Phys. B157, 45 (1979).

[31] A. Bassetto, M. Ciafaloni, and G. Marchesini, Nucl. Phys. B163, 477 (1980); Phys. Rep. 100, 203 (1983).

[32] M. Bengtsson and T. Sjöstrand, Z. Phys. C 37, 465 (1988).

[33] Yu. L. Dokshitzer, Sov. Phys. JETP 46, 641 (1977); G. Altarelli and G. Parisi, Nucl. Phys. B126, 298 (1977).

[34] G. Marchesini and B. R. Webber, Nucl. Phys. B310, 461 (1988); B349, 617 (1991).

[35] T. D. Gottschalk, Nucl. Phys. B277, 100 (1986); M. Bengtsson, T. Sjöstrand, and M. van Zijl, Z. Phys. C 32, 67 (1986).

[36] G. Marchesini and B. R. Webber, Nucl. Phys. B238, 1 (1984); B. R. Webber, Annu. Rev. Nucl. Part. Sci. 36, 253 (1986).

[37] D. Amati and G. Veneziano, Phys. Lett. 83B, 87 (1979); D. Amati, A. Bassetto, M. Ciafaloni, G. Marchesini, and G. Veneziano, Nucl. Phys. B173, 429 (1980).

[38] B. R. Webber, Nucl. Phys. B238, 492 (1984).

[39] R. Hagedorn, Thermodynamics of Strong Interactions, Cargèse, 1973, Lectures in Physics Vol. 6 (unpublished).

[40] UA5 Collaboration, G. J. Alner et al., Nucl. Phys. B291, 445 (1987).

[41] K. Goulianos, Phys. Rep. 101, 169 (1983).

[42] N. Pavel, Report No. DESY-95-147 1995 (unpublished).

[43] Ya. I. Azimov, Yu. L. Dokshitzer, V. A. Khoze, and S. I. Troyan, Z. Phys. C 27, 65 (1985); Yu. L. Dokshitzer, V. A. Khoze, and S. I. Troyan, ibid. 55, 107 (1992).

[44] ZEUS Collaboration, N. Pavel, in International Workshop on Deep Inelastic Scattering, Rome, Italy, 1996 (unpublished).

[45] ZEUS Collaboration, M. Derrick et al., Z. Phys. C 70, 391 (1996).

[46] B. Andersson, G. Gustafson, G. Ingelman, and T. Sjöstrand, Phys. Rep. 97, 33 (1983).

[47] A. H. Mueller, Phys. Rev. D 4, 150 (1971). 